\title{
Plastic deformations in crystal, polycrystal, and glass in binary mixtures under shear: Collective yielding
}

\author{
Hayato Shiba \\ Institute for Solid State Physics, University of Tokyo, Chiba 277-8581, Japan and \\ Department of Physics, Kyoto University, Kyoto 606-8502, Japan \\ Akira Onuki \\ Department of Physics, Kyoto University, Kyoto 606-8502, Japan
}

(Dated: July 27, 2021)

\begin{abstract}
Using molecular dynamics simulation, we examine the dynamics of crystal, polycrystal, and glass in a Lennard-Jones binary mixture composed of small and large particles in two dimensions. The crossovers occur among these states as the composition $c$ is varied at fixed size ratio. Shear is applied to a system of 9000 particles in contact with moving boundary layers composed of 1800 particles. The particle configurations are visualized with a sixfold orientation angle $\alpha_{j}(t)$ and a disorder variable $D_{j}(t)$ defined for particle $j$, where the latter represents the deviation from hexagonal order. Fundamental plastic elements are classified into dislocation gliding and grain boundary sliding. At any $c$, large-scale yielding events occur on the acoustic time scale. Moreover, they multiply occur in narrow fragile areas, forming shear bands. The dynamics of plastic flow is highly hierarchical with a wide range of time scales for slow shearing. We also clarify the relationship between the shear stress averaged in the bulk region and the wall stress applied at the boundaries.
\end{abstract}

PACS numbers: 83.10.Bb, 62.20.F-,61.43.-j, 61.72.-y

\section{INTRODUCTION}

A wide variety of rheological problems are well known in crystal, polycrystal, and glass, where plastic deformations are induced by structural changes with increasing applied stress [1, 2]. In crystal, dislocations play a major role in plasticity $[3] 6$. They appear and grow around relatively fragile objects such as point defects, preexisting dislocations, and grain boundaries. Viscoplastic deformations under applied stress then involve the motion of a large number of interacting dislocations. Furthermore, in multi-phase alloys with domain structures or precipitates, dislocations can appear at the interfaces to grow into softer regions $2,3,7,8$. In polycrystal, plastic deformations can also be induced by sliding motions of the particles at grain boundaries 9, as studied by molecular dynamics (MD) simulations [10 15]. In crystal and polycrystal, plastic events take place as bursts or avalanches spanning wide ranges of space and time scales as observed in acoustic emission experiments 4 and by transmission electron microscopy [5].

Much attention has also been paid to rheology in structurally disordered systems, including supercooled liquids and glass $16+28$, foam and microemulsion systems [29 31, colloid suspensions [32, 33, and granular materials 34 39. We mention an early experiment on glass-forming fluids by Simmons et al [16], who found strong shear-thinning behavior of the viscosity expressed as $\eta(\dot{\gamma}) \cong \eta(0) /\left(1+\dot{\gamma} \tau_{\eta}\right)$ in soda-lime-silica glasses under shear with rate $\dot{\gamma}$. Here $\tau_{\eta}$ is a rheological time of the order of the structural relaxation time $\tau_{\alpha}$, where $\tau_{\alpha}$ grows strongly from a microscopic to macroscopic time as the glass transition is approached [40]. This behavior has been reproduced in subsequent MD simulations (mostly in another expression $\eta \sim \dot{\gamma}^{-a}$ with $a \sim 0.8$ ). In molecular glasses this nonlinear regime emerges for $\dot{\gamma} \tau_{\alpha}>1$, where shear accelerates the rearrangement of particle configurations in jammed states [17. As a closely related problem, understanding of mechanical properties of amorphous metals at high strains is of great technological importance in metallurgy [1, 2, 41,47.

Numerous MD simulations on quiescent binary particle systems [48 59] revealed that the glass dynamics is highly heterogeneous on mesoscopic spatial scales. In model amorphous alloys, Takeuchi et al.48] observed mesoscopic heterogeneity in atomic motions in an applied strain. After early findings by Muranaka and Hiwatari [49] and by Harrowell and coworkers [50, Yamamoto and one of the present authors 51] examined breakage of appropriately defined bonds and identified relatively active regions. The broken bonds accumulated in long time intervals are heterogeneous such that their structure factor may be fitted to the Ornstein-Zernike form $\propto 1 /\left(1+k^{2} \xi^{2}\right)$, where the wave number $k$ is smaller than the inverse particle size and the correlation length $\xi$ grows with lowering the temperature $T$. Glotzer et al. [52 54 pointed out relevance of stringlike clusters of mobile particles whose lengths increase at low temperatures. Afterwards, some authors have claimed the presence of correlation between the structural heterogeneity in the particle configurations and the dynamic heterogeneity on long time scales [57 59]. Recently significant heterogeneity has been found in the elastic moduli in glass 60, 61, which is the origin of nonaffine elastic displacements for very small strains.

In glass under shear, the dynamic heterogeneity from the bond breakage becomes short-ranged as if a sheared state is mapped onto a quiescent state at a higher 
temperature [17, 18. However, some MD simulations on glass [20, 25, 27] realized organization of "shear bands" with localization of velocity gradients 62 on the system-size scale. Such bands were along the flow direction in Refs. [20, 25], while Furukawa et al. 27] realized shear bands transiently (with finite life times) equally in the flow and velocity-gradient directions in 2D using the Lees-Edwards boundary condition. In experiments on amorphous solids at low $T$, shear bands have been observed under uniaxial stress above a yield stress [1, 2, 44, 63, 64. The width of shear bands is microscopic in the initial stage 63. but can grow into micrometer sizes. Shear bands under uniaxial stress were realized in MD simulations [65 68] and in simulations of 2D phenomenological models 69, 70, where the band lines (or planes in 3D) make an angle of $\pi / 4$ with respect to the uniaxial direction.

In this paper, we will present MD simulations extending those in our previous papers 14, 58. We will examine the crossover among crystal, polycrystal, and glass with varying the composition $c$ in a model $2 \mathrm{D}$ binary mixture with shear. Here the temperature $T$ and the size ratio of the diameters of the two components $\sigma_{2} / \sigma_{1}$ are fixed. Further detailed discussions on the crossover with varying $c$ will be given elsewhere [71. We shall see that plastic events tend to take place over wide areas in short times also in glass, whereas some phenomenological theories were based on the assumption that plastic events are spatially localized in glass due to the structural disorder [41 43]. It is worth noting that some simulations have recently been performed on the size distribution of extended plastic events in glass 28. One of our purposes in this paper is to unambiguously visualize the formation and growth of plastic deformations over a wide range of time scales in a sufficiently large system.

The organization of this paper is as follows. In Sec.II, our model and our simulation method will be explained. In Sec.III, numerical results will be presented on nonlinear rheology with large stress drops and collective yielding on various time scales. We will also clarify the relationship between the applied wall stress and the shear stress averaged within the bulk region.

\section{MODEL AND SIMULATION METHOD}

We treat two-dimensional (2D) binary mixtures composed of two atomic species 1 and 2, as in our previous papers 14, 58. The particles interact via truncated Lennard-Jones (LJ) potentials,

$$
v_{\alpha \beta}(r)=4 \epsilon\left[\left(\frac{\sigma_{\alpha \beta}}{r}\right)^{12}-\left(\frac{\sigma_{\alpha \beta}}{r}\right)^{6}\right]-C_{\alpha \beta}
$$

which are characterized by the energy $\epsilon$ and the interaction lengths $\sigma_{\alpha \beta}=\left(\sigma_{\alpha}+\sigma_{\beta}\right) / 2(\alpha, \beta=1,2)$. Here $\sigma_{1}$ and $\sigma_{2}$ represent the soft-core diameters of the two components and their ratio is fixed at $\sigma_{2} / \sigma_{1}=1$.4. For $r>r_{\text {cut }}=3.2 \sigma_{1}$, we set $v_{\alpha \beta}=0$ and the constant $C_{\alpha \beta}$ ensures the continuity of $v_{\alpha \beta}$ at $r=r_{\text {cut }}$. The mass ratio is fixed at $m_{1} / m_{2}=\left(\sigma_{1} / \sigma_{2}\right)^{2}$.

We divide the system into three regions (see Fig. 6 below). In the bulk region $-0.5 L<x, y<0.5 L$, we initially placed $N=N_{1}+N_{2}=9000$ particles. The composition of the larger particles is defined as

$$
c=N_{2} /\left(N_{1}+N_{2}\right) .
$$

The volume $L^{2}$ of the bulk region is chosen such that the volume fraction of the soft-core regions is fixed at 1 or 72 .

$$
\phi=\left(N_{1} \sigma_{1}^{2}+N_{2} \sigma_{2}^{2}\right) / L^{2}=1 .
$$

For example, $L=97.12$ for $c=0.05$ and $L=103.58$ for $c=0.2$. Thus $L \sim 100 \sigma_{1}$ in our simulation. We apply shear flow by the boundary motions of two boundary layers, which are expressed by $-0.6 L<y<-0.5 L$ and $-0.5 L<x<0.5 L$ at the bottom and by $0.5 L<y<$ $0.6 L$ and $-0.5 L<x<0.5 L$ at the top. In each layer, $N_{b}=900$ binary particles with the same composition and size ratio were initially placed. They are attached to it by the spring potential,

$$
u_{j}\left(\boldsymbol{r}-\boldsymbol{R}_{j}\right)=\frac{1}{2} K\left|\boldsymbol{r}-\boldsymbol{R}_{j}\right|^{2},
$$

where $\boldsymbol{R}_{j}$ are pinning points appropriately determined in the boundary layers (see below). The spring constant is set equal to $K=20 \epsilon \sigma_{1}^{-2}$. These bound particles also interact with the neighboring bound and unbound particles with the common Lennard-Jones potentials in Eq. (1). The total potential energy is thus written as

$$
U=\sum_{j, k \in \text { all }} \phi_{j k}+\sum_{j \in b} u_{j}
$$

The $\phi_{j k}=v_{\alpha \beta}\left(\left|\boldsymbol{r}_{j}-\boldsymbol{r}_{k}\right|\right)$ is the pair potential between the particles $j$ and $k$, with $j$ and $k$ being either unbound or bound to the walls. The $u_{j}$ is the spring potential in Eq.(4) between the bound particle $j \in b$ and the pinning point $\boldsymbol{R}_{j}$. After application of shear flow at $t=0$ the pinning points depend on time as

$$
\boldsymbol{R}_{j}(t)=\boldsymbol{R}_{j}(0) \pm \frac{1}{2} L \dot{\gamma} t \boldsymbol{e}_{x},
$$

where $\boldsymbol{e}_{x}$ is the unit vector along the $x$ axis. If its $x$ component $X_{j}(t)$ became larger than $L / 2$ in the upper layer (smaller than $-L / 2$ in the lower layer), it was decreased (increased) by $L$. That is, we assumed the periodic boundary condition along the $x$ axis. In our simulation the unbound particles rarely penetrated into the boundary layers deeper than $\sigma_{1}$.

We integrated the equations of motion using the leapfrog algorithm under the periodic boundary condition along the $x$ axis. The time step of integration is $0.002 \tau$ with

$$
\tau=\sigma_{1} \sqrt{m_{1} / \epsilon}
$$


The unbound particles obeyed the Newton equations $m_{j} \ddot{\boldsymbol{r}}_{j}=-\partial U / \partial \boldsymbol{r}_{j}$, where $\ddot{\boldsymbol{r}}_{j}=d^{2} \boldsymbol{r}_{j} / d t^{2}$. To subtract the heat produced in shear flow, we attached a NosèHoover thermostat [73, 74] to each boundary layer. That is, the bound particles $j \in \mathcal{B}$ are governed by

$$
m_{j} \ddot{\boldsymbol{r}}_{j}=-\frac{\partial U}{\partial \boldsymbol{r}_{j}}-\zeta_{\mathcal{B}} m_{j}\left(\dot{\boldsymbol{r}}_{j}-\boldsymbol{v}_{\mathcal{B}}\right)
$$

where $\dot{\boldsymbol{r}}_{j}=d \boldsymbol{r}_{j} / d t, \mathcal{B}$ represents the top or bottom boundary, and $\boldsymbol{v}_{\mathcal{B}}\left(= \pm(L \dot{\gamma} / 2) \boldsymbol{e}_{x}\right)$ is the boundary velocity at the top or bottom. The two thermostat variables $\zeta_{\text {bot }}(t)$ and $\zeta_{\text {top }}(t)$ obeyed

$$
\frac{d}{d t} \zeta_{\mathcal{B}}=\frac{1}{\tau_{\mathrm{NH}}^{2}}\left[\frac{1}{N_{b} k_{B} T} \sum_{j \in \mathcal{B}} \frac{m_{j}}{2}\left|\dot{\boldsymbol{r}}_{j}-\boldsymbol{v}_{\mathcal{B}}\right|^{2}-1\right],
$$

where $\tau_{\mathrm{NH}}$ is the thermostat characteristic time. We set $\tau_{\mathrm{NH}}=0.304 \tau$ in this paper. Then the local temperature (the local particle kinetic energy) became nearly homogeneous in the bulk region during plastic flow with shear $\dot{\gamma}=10^{-4} \tau^{-1} 75$. Here the thermal diffusion time was shorter than the inverse shear $1 / \dot{\gamma}$.

We explain how we prepared the initial particle configurations to which a shear flow was applied. (i) We first equilibrated the bulk and boundary regions independently without their mutual interactions. The particles in these regions interacted via the Lennard-Jones potentials (1) in a liquid state at $T=2 \epsilon / k_{B}$ in a time interval of $10^{3} \tau$ under the periodic boundary condition in the $x$ and $y$ axes. (ii) Then we quenched the system to $T=0.2 \epsilon / k_{B}$ and further equilibrated the system for a time interval of $10^{3} \tau$. After this low-temperature equilibration, we chose the particle positions in the boundary layers as the initial pinning points $\boldsymbol{R}_{j}(0)$ in Eq. (4) and introduced the spring potential (4) of the bound particles, the LJ potentials (1) between the bound and unbound particles, and the Nosè-Hoover thermostats of the boundary layers. (iii) We further waited for time interval of $5 \times 10^{3} \tau$ until we detected no appreciable time evolution in various thermodynamic quantities. After this second low-temperature equilibration, we applied a shear flow by sliding the pinning points as in Eq.(6).

\section{SIMULATION RESULTS}

Hereafter the space coordinates $x$ and $y$, the time $t$, the shear rate $\dot{\gamma}$, and the temperature $T$ will be measured in units of $\sigma_{1}, \tau, \tau^{-1}$, and $\epsilon / k_{B}$, respectively, while shear stresses will be in units of $\epsilon \sigma_{1}^{-2}$. We will treat high-density sheared states at $T=0.2$ and $\dot{\gamma}=10^{-4}$. The boundary speed $(\sim 0.005)$ is much slower than the thermal velocity $(\sim 0.6)$. There is no tendency of phase separation as in our previous work [14, 58.

\section{A. Previous results}

Jammed particle configurations in binary particle systems are very complicated depending on $c$ in Eq. $(2), \phi$ in Eq.(3), $T$, and $\sigma_{2} / \sigma_{1}$. In our previous work [58, 71, we have examined this problem with varying $c$ without and with shear. We here explain some characteristic features in the crossover among crystal, polycrystal, and glass for $\sigma_{2} / \sigma_{1}=1.4$ and $T=0.2$.

For small $c$ or $1-c$ less than a critical composition of order 0.05 , the overall crystalline order is attained, where the particles of the minority species form localized defects or isolated clusters with various sizes. For $0.05 \lesssim c \lesssim 0.15$ or $0.8 \lesssim c \lesssim 0.95$, polycrystal states are realized, where grain boundaries composed of the two species enclose crystalline domains consisting of the majority species. With increasing $c$ or $1-c$, the grain boudaries are gradually thickened into percolated amorphous layers enclosing small crystalline domains. If $c$ and $1-c$ are not small, glass states are eventially realized.

In polycrystal between crystal and glass, the grain boundary motions are severely slowed down in the presence of size dispersity $\sigma_{2} / \sigma_{1} \neq 1$, while the grain boundary motions are rapid in one-component systems [76. As compared to the particles within the crystalline regions, those in the grain boundary regions are relatively mobile and their collective motions give rise to the dynamic heterogeneity on long time scales [15, 58. Thus the origin of the dynamic heterogeneity is rather clear in polycrystal. Since small crystalline regions still remain in glass, the glass dynamics can be understood as the small-grain-size limit of the polycrystal dynamics [15, 58 . Also varying the degree of disorder, Kawasaki et al. [59] claimed that such remaining "medium-range crystalline order" controls both the ease of vitrification and nature of the glass transition.

The structural relaxation time $\tau_{\alpha}$ without shear was of order $10^{4}$ in glass, was longer in polycrystal, and tended to infinity in crystal from the decay of the self-timecorrelation function [58. The grain boundary motions are much suppressed in the presence of size dispersity.

\section{B. Orientation angle $\alpha_{j}$, disorder variable $D_{j}$, and bond breakage}

We introduce methods and techniques of detecting and visualizing structural disorder and dynamic heterogeneities [14, 17, 51, 58. In our 2D systems a large fraction of the unbound particles are enclosed by six particles. The local crystalline order may then be represented by a sixfold orientation [77. We define an orientation angle $\alpha_{j}$ in the range $-\pi / 6 \leq \alpha_{j}<\pi / 6$ for each unbound particle $j$ using the complex number,

$$
\begin{aligned}
\Psi_{j} & =\sum_{k \in \text { bonded }} \exp \left[6 i \theta_{j k}\right] \\
& =\left|\Psi_{j}\right| e^{6 i \alpha_{j}} .
\end{aligned}
$$



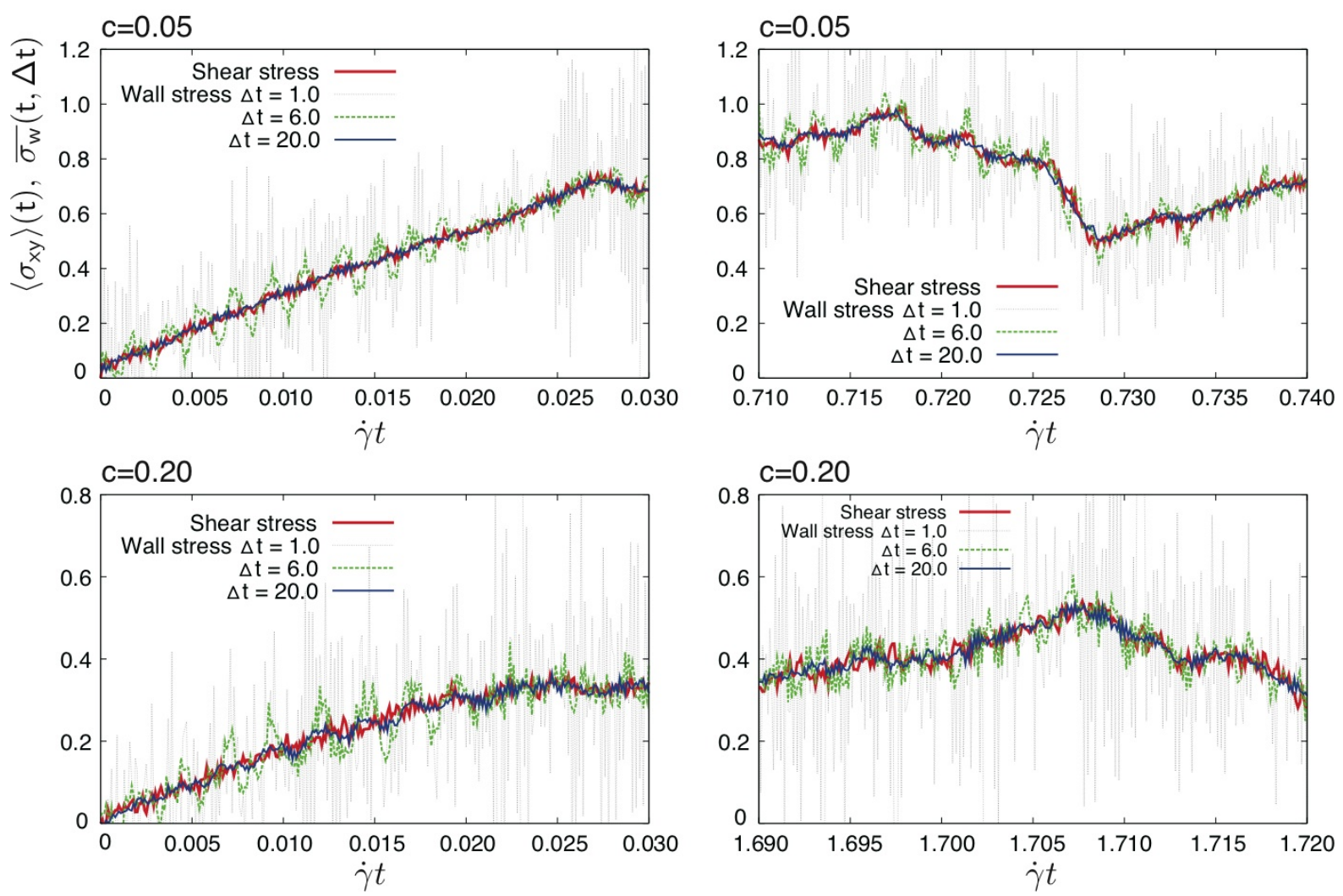

FIG. 1: (Color online) Smoothed wall stress $\bar{\sigma}_{\mathrm{w}}(t, \Delta t)$ in Eq. 17 and average stress $\left\langle\sigma_{x y}\right\rangle(t)$ under shear $\dot{\gamma}=10^{-4}$ in the initial stage (left) and in the plastic flow regime (right), where $c=0.05$ (top) and $c=0.2$ (bottom) with $\Delta t=1,6$, and 20 .

In the first line, the summation is over the particles bonded to the particle $j$. In our case, the two particles $j \in \alpha$ and $k \in \beta$ are bonded if their distance $r_{j k}=\left|\boldsymbol{r}_{j}-\boldsymbol{r}_{k}\right|$ is shorter than $1.5 \sigma_{\alpha \beta}$. The $\theta_{j k}$ is the angle of the relative vector $\boldsymbol{r}_{j}-\boldsymbol{r}_{k}$ with respect to the $x$ axis. The second line is the definition of $\alpha_{j}$. It is convenient to introduce another non-negative-definite variable representing the degree of disorder or the deviation from hexagonal order for each particles $j$ by

$$
\begin{aligned}
D_{j} & =\sum_{k \in \text { bonded }}\left|e^{6 i \alpha_{j}}-e^{6 i \alpha_{k}}\right|^{2} \\
& =2 \sum_{k \in \text { bonded }}\left[1-\cos 6\left(\alpha_{j}-\alpha_{k}\right)\right] .
\end{aligned}
$$

For a perfect crystal at low temperature this quantity arises from thermal vibrations and is nearly zero, but for particles around defects it assumes large values in the range 15-20.

In jammed states, particle configuration changes can be conveniently visualized by using the method of bond breakage. That is, for each particle configuration at a time $t$, a pair of particles $i \in \alpha$ and $j \in \beta$ is considered to be bonded if

$$
r_{i j}(t)=\left|\boldsymbol{r}_{i}(t)-\boldsymbol{r}_{j}(t)\right| \leq A_{1} \sigma_{\alpha \beta},
$$

where $\sigma_{\alpha \beta}=\left(\sigma_{\alpha}+\sigma_{\beta}\right) / 2$. We set $A_{1}=1.2$; then, $A_{1} \sigma_{\alpha \beta}$ is slightly larger than the peak distance of the pair correlation functions $g_{\alpha \beta}(r)$. After a time interval $\Delta t$, the bond is regarded to be broken if

$$
r_{i j}(t+\Delta t) \geq A_{2} \sigma_{\alpha \beta}
$$

where we set $A_{2}=1.5$. In the following figures, bonds broken during a time interval $\left[t_{1}, t_{2}\right]$ will be marked by $\times$ at the middle point $\frac{1}{2}\left(\boldsymbol{r}_{i}\left(t_{2}\right)+\boldsymbol{r}_{j}\left(t_{2}\right)\right)$ of the two particle positions at the terminal time $t_{2}$. The broken bond number in the system in the time interval $[t, t+\Delta t]$ will be denoted by $\Delta N_{b}(t)$.

\section{Average stress and wall stress}

In the literature of MD simulations of fluids, the following shear stress has been calculated [79]:

$$
\left\langle\sigma_{x y}\right\rangle(t)=\frac{-1}{L^{2}}\left[\sum_{j} m_{j} \dot{x}_{j} \dot{y}_{j}-\sum_{j k} \frac{x_{j k} y_{j k}}{2 r_{j k}} \phi_{j k}^{\prime}\right] .
$$

where we sum over the unbound particles $j$ and $k$. Here $\dot{x}_{j}=d x_{j} / d t$ and $\dot{y}_{j}=d y_{j} / d t$ represent the velocity components, $\phi_{j k}=v_{\alpha \beta}\left(r_{j k}\right)(j \in \alpha, k \in \beta)$ is the pair potential between $j$ and $k$, and $\phi_{j k}^{\prime}=\partial \phi_{j k} / \partial r_{j k}$, with $\boldsymbol{r}_{j}-\boldsymbol{r}_{k}=\left(x_{j}-x_{k}, y_{j}-y_{k}\right)$ and $r_{j k}=\left|\boldsymbol{r}_{j}-\boldsymbol{r}_{k}\right|$. This 
(a) $\mathrm{C}=0.02$

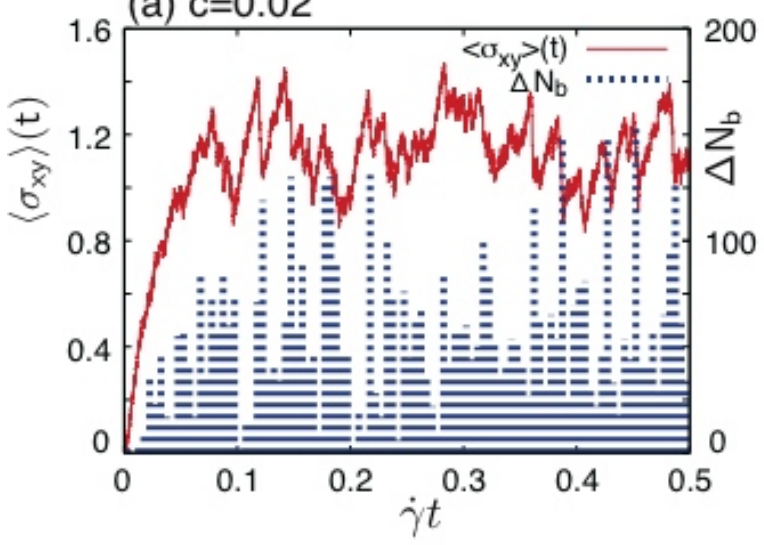

(c) $\mathrm{C}=0.10$

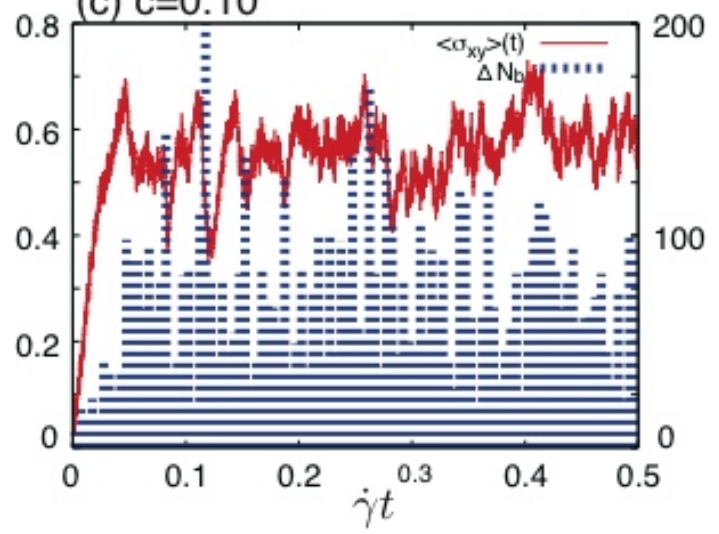

(b) $\mathrm{c}=0.05$

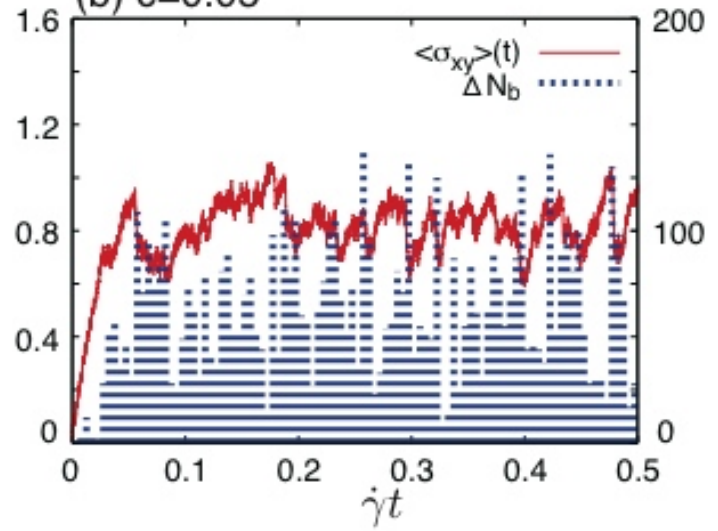

(d) $\mathrm{C}=0.20$

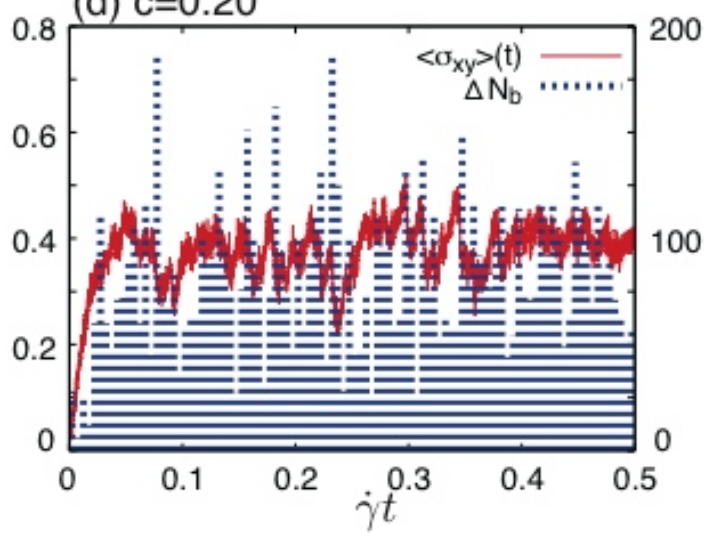

FIG. 2: (Color online) Red solid lines (left scale): Average shear stress $\left\langle\sigma_{x y}\right\rangle(t)$ (in units of $\epsilon \sigma_{1}^{-2}$ ) vs strain $\dot{\gamma} t$ after application of shear $\dot{\gamma}=10^{-4}$ for (a) $c=0.02$, (b) 0.05 , (c) 0.10 , and (d) 0.20 . Blue dotted bars (right scale): broken bond number $\Delta N_{b}(t)$ in time interval with width $50=5 \times 10^{-3} / \dot{\gamma}$ in the same runs. Large stress drops are accompanied by large $\Delta N_{b}(t)$.

quantity is the minus of the space average of the $x y$ component of the stress tensor $\Pi_{x y}(\boldsymbol{r}, t)$ contributed by the unbound particles.

However, it is not clear how $\left\langle\sigma_{x y}\right\rangle(t)$ in Eq. (14) is related to the experimental shear stress. In our geometry, $\left\langle\sigma_{x y}\right\rangle(t)$ may be related to the forces to the fluid exerted by the top and bottom boundary layers via the springs,

$$
\begin{aligned}
& F_{\text {top }}(t)=K \sum_{j \in \text { top }}\left[X_{j}(t)-x_{j}\right], \\
& F_{\text {bot }}(t)=-K \sum_{j \in \text { bot }}\left[x_{j}-X_{j}(t)\right],
\end{aligned}
$$

where the particle $j$ is bound to the top layer in $F_{\text {top }}(t)$ and to the bottom layer in $F_{\text {bot }}(t)$ with $X_{j}(t)=X_{j}(0) \pm$ $\dot{\gamma} t L / 2$ being the $x$ component of the pinning position $\boldsymbol{R}_{j}(t)$ in Eq. (6). In shear flow, the time-dependent energy input from the walls to the fluid is given by

$$
\dot{W}(t)=L^{2} \dot{\gamma} \sigma_{\mathrm{w}}(t)
$$

per unit time. The $\sigma_{\mathrm{w}}(t)$ is the wall stress written as

$$
\sigma_{\mathrm{w}}(t)=\left[F_{\text {top }}(t)-F_{\text {bot }}(t)\right] / 2 L \text {. }
$$

The total particle energy $E=\sum_{j} m_{j} \dot{\boldsymbol{r}}_{j}^{2} / 2+U$ changes in time as

$$
\frac{d}{d t} E=\dot{W}-\sum_{\mathcal{B}} \sum_{j \in \mathcal{B}} \zeta_{\mathcal{B}} m_{j}\left|\dot{\boldsymbol{r}}_{j}-\boldsymbol{v}_{\mathcal{B}}\right|^{2}
$$

where the second term in the right hand side represents the energy absorption in the boundary layers.

In steady states, the time averages of $\sigma_{\mathrm{w}}(t)$ and $\left\langle\sigma_{x y}\right\rangle(t)$ should coincide. This time-averaged value $\bar{\sigma}_{\mathrm{w}}=$ $\bar{\sigma}_{x y}$ is equal to the steady-state shear viscosity $\eta(\dot{\gamma})$ multiplied by $\dot{\gamma}$. However, $\sigma_{\mathrm{w}}(t)$ consists of rapid motions of the spring contraction and extension on the time scale of

$$
\tau_{\mathrm{sp}}=\pi\left(m_{1} / K\right)^{1 / 2}
$$

which is of order $\tau$ in Eq.(7). In Fig. 1, we compare $\left\langle\sigma_{x y}\right\rangle(t)$ in Eq. (14) and a smoothed wall-stress defined by

$$
\bar{\sigma}_{\mathrm{w}}(t, \Delta t)=\frac{1}{\Delta t} \int_{-\Delta t / 2}^{\Delta t / 2} d t^{\prime} \sigma_{\mathrm{w}}\left(t+t^{\prime}\right),
$$

in the initial stage and in the plastic flow regime. This quantity should coincide with $\left\langle\sigma_{x y}\right\rangle(t)$ for long-time 
smoothing in the case $\Delta t>\tau_{\mathrm{st}}$, where $\tau_{\mathrm{st}}$ is a crossover time of the stress response. In our case, we find $\tau_{\mathrm{st}} \sim 20 \tau$. We notice that this $\tau_{\mathrm{st}}$ is of the order of the acoustic traversal time,

$$
\tau_{\mathrm{ac}}=L / c_{\perp},
$$

where $c_{\perp}=(G / \bar{\rho})^{1 / 2}$ is the transverse sound velocity. Here the average mass density $\bar{\rho}=\left[m_{1}(1-c)+m_{2} c\right] N / L^{2}$ is of order $m_{1} \sigma_{1}^{-2}$ and the shear modulus $G$ will be calculated to be of order $20 \epsilon \sigma_{1}^{-2}$ in Figs. 2 and 3 below, so $c_{\perp} \sim 5 \sigma_{1} / \tau$ and $\tau_{\mathrm{ac}} \sim 20 \tau$. The stress deviations arising from local plastic deformations propagate outwards with sppeds on the order of the sound velocity, though they are not small elastic deformations. In our simulation, we numerically find the following approximate relation,

$$
\sigma_{\mathrm{w}}(t)-\left\langle\sigma_{x y}\right\rangle(t) \cong \frac{1}{L^{2}} \frac{d}{d t} \sum_{j \in \text { all }} m_{j} y_{j} \dot{x}_{j},
$$

where all the unbound and bound particles are summed in the right hand side. See the appendix for more details leading to Eq. (22).

Furthermore, we consider the consequence of the energy balance Eq. (18) in steady states, where the time average of $d E / d t$ vanishes. In the right hand side of Eq.(18), the time average of $\dot{W}$ is equal to $L^{2} \dot{\gamma} \bar{\sigma}_{x y}$ in terms of the average stress $\bar{\sigma}_{x y}$ (the time average of $\left.\left\langle\sigma_{x y}\right\rangle(t)\right)$, while those of $\zeta_{\text {top }}$ and $\zeta_{\text {bot }}$ should assume the same value $\bar{\zeta}=\overline{\zeta_{\text {top }}}=\overline{\zeta_{\text {top }}}$. The kinetic energies $\sum_{j \in \mathcal{B}} m_{j}\left|\dot{\boldsymbol{r}}_{j}-\boldsymbol{v}_{\mathcal{B}}\right|^{2} / 2$ of the bound particles fluctuated with amplitude of order $5 \%$ of the mean value $N_{b} k_{B} T$ in our simulation. Thus,

$$
\bar{\zeta}=L^{2} \dot{\gamma} \bar{\sigma}_{x y} / 4 N_{b} k_{B} T
$$

where the numerator in the right hand side is the viscous heat production. In our simulation, this balance was well achieved and the temperature was kept nearly homogeneous in the plastic flow regime [75]. For example, for $c=0.05$, we obtain almost the same time averages $\overline{\zeta_{\text {top }}}=0.0010$ and $\overline{\zeta_{\text {top }}}=0.0011$, while the right hand side of Eq.(23) is 0.0011 using $\bar{\sigma}_{x y}=0.855 \mathrm{in} \mathrm{Fig.3.} \mathrm{The} \mathrm{ther-}$ mostat variables $\zeta_{\text {top }}(t)$ and $\zeta_{\text {bot }}(t)$ undergo large temporal fluctuations with amplitude of order 0.1 for this case. In equilibrium, the thermostatic variable $\zeta(t)$ (usually attached to all the particles under the periodic boundary condition) fluctuates around zero [73, 74].

\section{Nonlinear rheology: plastic and elastic strains}

In Fig. 2, we show the average shear stress $\left\langle\sigma_{x y}\right\rangle(t)$ and the broken bond numbers $\Delta N_{b}(t)$ as functions of the strain $\dot{\gamma} t$ after application of shear for $c=0.02,0.05,0.1$, and 0.2 . Here the time average and variance of $\left\langle\sigma_{x y}\right\rangle(t)$ decrease with increasing $c$. In the initial regime $\dot{\gamma} t \lesssim$ 0.04 , it increases linearly as

$$
\left\langle\sigma_{x y}\right\rangle(t) \cong G \dot{\gamma} t .
$$

This is the definition of the shear modulus $G$ in this paper. In glass, it should be considerably smaller than the infinite-frequency shear modulus $G_{\infty}$ introduced by Zwanzig and Mountain [78, since the correlationfunction expression for $G_{\infty}$ is based on the assumption of affine deformations of the particle positions (see the appendix of Chapter I of Ref. [79]). However, in glass, the local elastic moduli are highly inhomogeneous and the particle displacements are strongly nonaffine even for very small strains [60, 61]. In our simulation $G_{\infty} / G=2-3$ is obtained in polycrystal and glass. The expression for the transverse sound velocity $c_{\perp}=(G / \bar{\rho})^{1 / 2}$ is only approximate if use is made of $G$ in Eq.(24).

In the subsequent plastic flow in Fig. $2,\left\langle\sigma_{x y}\right\rangle(t)$ exhibits large temporal fluctuations in spite of the fact that it is the space average in the bulk region with volume $L^{2} \sim 10^{4}[28$, 80. The maximum stress drops are of order 0.2 for small $c$ and of order 0.1 at $c=0.2$. They sometimes take place abruptly on a time scale of the acoustic time $\tau_{\mathrm{ac}}$ in Eq.(21). They are induced by collective configuration changes of the particle positions as will be examined later. In Fig.2, this is demonstrated from the histograms of the broken bonds in time interval with width $50=5 \times 10^{-3} / \dot{\gamma}[51$. In MD simulations on amorphous systems, similar stress-strain curves have been calculated (see Refs. 23 25], for example).

In plastic flow, the typical amplitude of the stress fluctuations around the mean value is inversely proportional to the system length $L$ (to the square of the volume in 3D) [23, 24, 80]. In crystalline systems, the stress-strain curve becomes smooth for macroscopic samples, so that the avalanche behavior of the dislocation motions was detected by acoustic emission measurements using a piezoelectric transducer [4. In some polycrystalline dilute alloys such as Al-4 at.\% Mg, however, a noisy stress-strain curve has been observed even for macroscopic samples (the Portevin-Le Chatelier effect) 81.

We introduce the plastic and elastic strains by simple arguments. In time interval with width $\Delta t$, the broken bond number $\Delta N_{b}(t)$ and the plastic strain increment $\Delta \gamma_{\mathrm{pl}}$ are related as

$$
\Delta N_{b} \sim N \Delta \gamma_{\mathrm{pl}},
$$

where $N$ is the total unbound particle number. In the plastic flow regime, the average of $\Delta \gamma_{\mathrm{pl}}$ over many successive time intervals should be equal to the applied strain increment $\Delta \gamma=\dot{\gamma} \Delta t=5 \times 10^{-3}$. Thus the time average of $\Delta N_{b}$ is estimated as

$$
\overline{\Delta N_{b}} \sim N \dot{\gamma} \Delta t,
$$

which is consistent with Fig. 2 since the right hand side of Eq. (26) is 45. The elastic strain increment in each time interval is given by the difference,

$$
\Delta \gamma_{\mathrm{el}}=\dot{\gamma} \Delta t-\Delta \gamma_{\mathrm{pl}}
$$

In Fig. 2 when $\left\langle\sigma_{x y}\right\rangle(t)$ increases gradually, $\Delta N_{b}$ is relatively small and $\Delta \gamma_{\mathrm{el}}$ also increases gradually up to of 


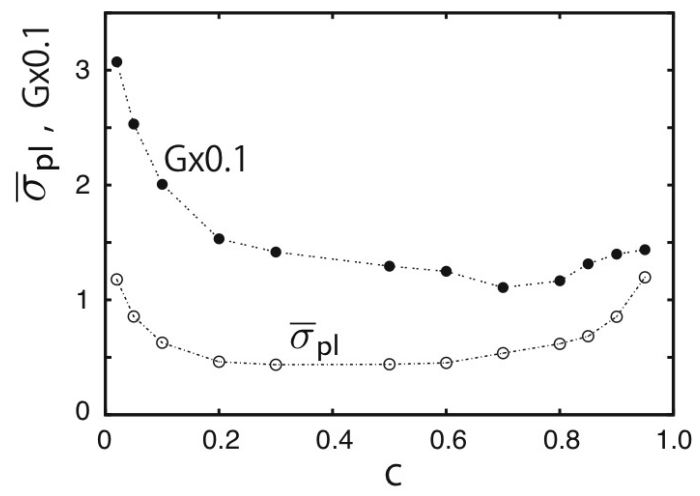

FIG. 3: Shear modulus $G$ in the initial stage in Eq. 24) and time-averaged stress $\bar{\sigma}_{x y}$ in units of $\epsilon \sigma_{1}^{-2}$ in the plastic flow regime in Eq. 29 as functions of $c$ at $\dot{\gamma}=10^{-4}$. The volume fraction is fixed as in Eq. (3).

order $\dot{\gamma} \Delta t$. On the other hand, when $\left\langle\sigma_{x y}\right\rangle(t)$ drops abruptly, $\Delta N_{b}$ is relatively large and $\Delta \gamma_{\mathrm{el}}$ is negative. As a result, the time average of the elastic strain increment $\Delta \gamma_{\mathrm{el}}$ should vanish, since the time average of the elastic strain $\gamma_{\mathrm{el}}(t)$ remains a constant $\bar{\gamma}_{\mathrm{el}}$ in plastic flow. Here we relate the average elastic strain $\bar{\gamma}_{\mathrm{el}}$ to the time average of the shear stress $\bar{\sigma}_{x y}$ as

$$
\bar{\gamma}_{\mathrm{el}} \sim \bar{\sigma}_{x y} / G
$$

In our case we define the time-averaged stress $\bar{\sigma}_{x y}$ as

$$
\bar{\sigma}_{x y}=\frac{1}{t_{2}-t_{1}} \int_{t_{1}}^{t_{2}} d t\left\langle\sigma_{x y}\right\rangle(t),
$$

with $t_{1}=0.2 / \dot{\gamma}$ and $t_{2}=1 / \dot{\gamma}$ in the plastic flow regime. In Fig. 3, we display the shear modulus $G$ in the initial stage defined by Eq. 24 and the time-averaged stress $\bar{\sigma}_{x y}$ in the plastic flow regime. For $c \lesssim 0.5$, we have $G / \bar{\sigma}_{x y} \sim 30$, which then leads to a reasonable estimate, $\bar{\gamma}_{\mathrm{el}} \sim 0.03$, from Eq.(28). For $c \gtrsim 0.5$ the ratio gradually decreases but remains larger than 10. Remarkably, as functions of $c$, both $G$ and $\bar{\sigma}_{x y}$ are minimum at intermediate compositions. Shikata et al. 82 measured the linear shear viscosity $\eta(c)$ of bimodal colloidal suspensions as a function of $c$ and found its minimum at intermediate $c$, where the colloid volume fraction was fixed.

\section{E. Fundamental deformation modes: dislocation gliding and grain boundary sliding}

In crystals containing defects and polycrystals composed of grains, plastic deformations under applied strain take place in two manners. First, dislocations formed around defects (grain boundaries, point defects, and preexisting dislocations) glide into the grain interior with a speed on the order of the sound velocity [83. They eventually form slip planes (lines in 2D) bounded by grain boundaries in polycrystals. Second, the particles at the
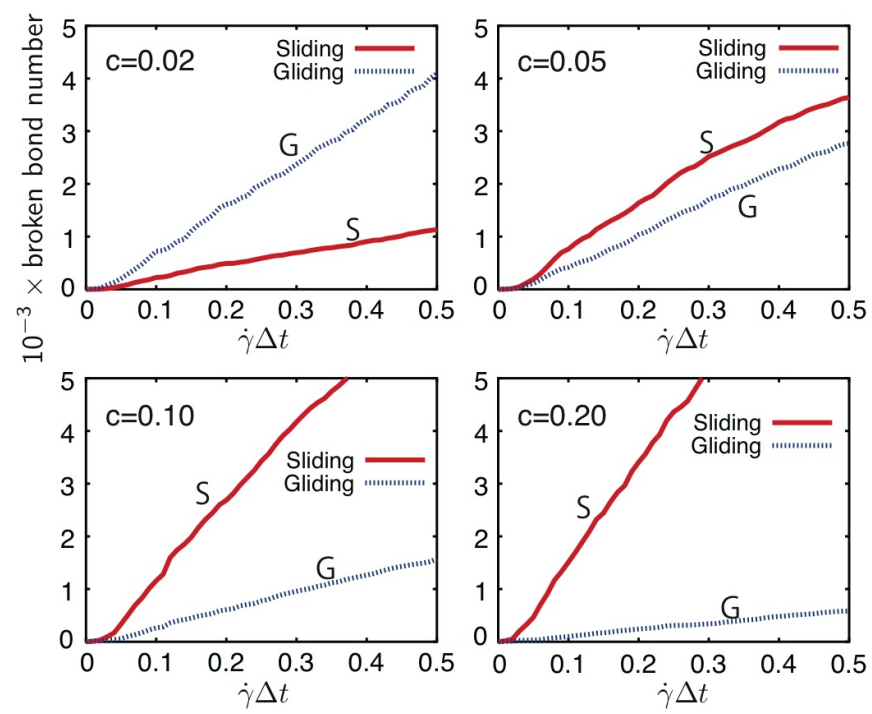

FIG. 4: (Color online) Accumulated numbers of broken bonds in a time interval with width $\Delta t$ vs strain increment $\dot{\gamma} \Delta t$ for $c=0.02,0.05,0.10$, and 0.20 . Broken bonds between particles $i$ and $j$ are classified into type $\mathrm{G}$ with $D_{i}+D_{j}>4$ (red solid lines) and type $\mathrm{S}$ with $D_{i}+D_{j}<4$ (blue dotted lines). Type $\mathrm{S}$ broken bonds increase with increasing disorder. (a) [3232:3236]

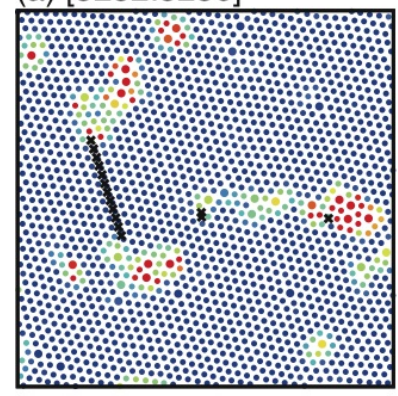

(c) $[3240: 3244]$

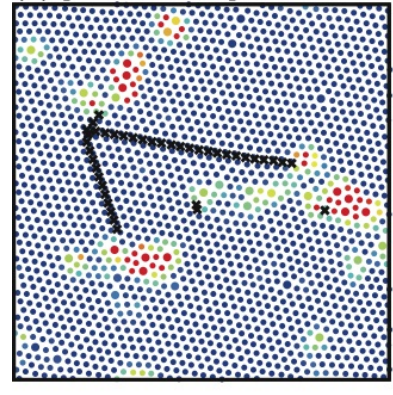

(b) [3236:3240]

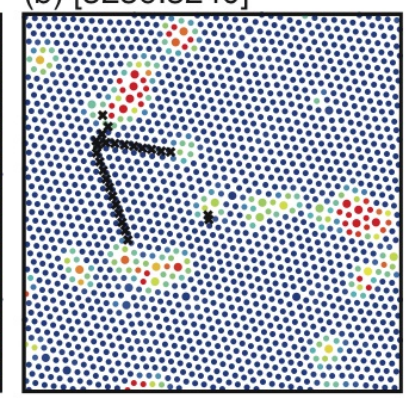

[3232:3240]

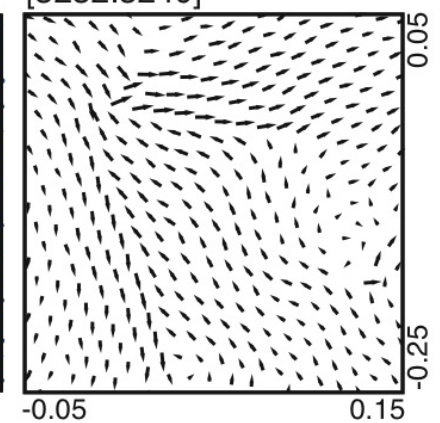

FIG. 5: (Color online) Successive snapshots of broken bonds $(\times)$ generated in the time intervals $[3232,3236]$ in (a), $[3236,3240]$ in (b), and $[3240,3244]$ in (c) in a crystal state at $c=0.02$ in the region $-0.1 L \leq x \leq 0.2 L$ and $0 \leq y \leq 0.3 L$. Each particle has a color depending on its value of the disorder variables $D_{j}$. Right bottom: Displacement vectors $\boldsymbol{r}_{j}\left(t_{2}\right)-\boldsymbol{r}_{j}\left(t_{1}\right)$ with $t_{1}=3232$ and $t_{2}=3240$ in the narrower region $-0.05 L \leq x \leq 0.15 L$ and $0.05 L \leq y \leq 0.25 L$ containing the two slip lines in panel (b). An edge dislocation is evident at the moving slip end. 
grain boundaries intermittently undergo sliding motions releasing elastic energies accumulated within the crystalline grains. In real 3D polycrystals 913 , the dislocation mechanism dominates for grain sizes larger than a critical size $d_{c} \sim 10 \mathrm{~nm}$, while the sliding mechanism dominates for smaller grain sizes.

In our previous 2D simulation with $N=1000$ [14, we observed sliding motions of the particles at the grain boundaries in polycrystal, causing large stress fluctuations. There, however, the sliding motions extended over the whole region due to the small system size. In the present work with $N=9000$, we observe these two deformation modes as local events in crystal and polycrystal, while the sliding motions become increasingly shortranged in glass.

In glass, the local crystalline order can be defined only over short distances. Therefore, fundamental plastic elements have been supposed to be quite localized in glass 41 43. In a 2D amorphous soap bubble raft, Argon and Kuo [84] observed nucleation of a dislocation pair giving rise to a small-scale slip, though such dislocations did not glide more than two to three bubble distances. With the size ratio $\sigma_{2} / \sigma_{1}$ being rather close to unity, Deng et al. 65. found extended slips in 2D simulation. In glass in our simulation also, short slips may be identified, where the slip length does not much exceed the size of the local crystal structure. However, we shall see (in Figs.12-14 below) that such slips successively appear in their neighborhood to form large-scale aggregates.

Figure 4 shows the numbers of broken bonds versus the strain increment $\dot{\gamma} \Delta t$ for $c=0.02,0.05,0.1$, and 0.2 , where $\Delta t$ is the time interval in Eq. (13). We divide the broken bonds into those with $D_{i}+\bar{D}_{j}>4$ (type $\mathrm{S}$ ) and those with $D_{i}+D_{j}<4$ (type G), where the bond between the particles $i$ and $j$ is broken. At small $c$, the bond breakage is mainly caused by the dislocation gliding between defects, where the resultant broken bonds are type G. In glass, most broken bonds are type $\mathrm{S}$ along boundaries of small crystalline regions.

Figure 5 displays a typical example of dislocation gliding along principal crystal axes in a crystal state with $c=0.02$. In the first panel (left top) a pair of edge dislocations appear around a defect and one of them glides into the crystal region forming a slip line until it is pinned at another defect. In the second panel (right top), another edge dislocation is gliding, whose Burgers vector is in the slip direction with magnitude being equal to the lattice constant $b$ of the hexagonal lattice [3. In the third one (left bottom) the second slip is also pinned at a point defect. In the right bottom panel, the displacements of the particles are around the two slips in the panel (b).

From many such runs we find that most slips extend nearly along the crystal axes when they become nearly parallel to the $x$ or $y$ axis under slow shearing. We also notice that there can be two types of slips depending on the directions of the Burgers vectors at the slip ends. That is, the particle displacements around a slip are either clockwise (type C) or counterclockwise (type CC).
In our simulation, slips along the $x$ axis are type $\mathrm{C}$ and those along the $y$ are type CC. See the right bottom panel of Fig.4 and the figures in Ref.[70] as examples.

We claim that the preferred directions of the growth of plastic deformations should be determined by the angledependence of the elastic energy. For example, let us assume the presence of a slip with length $\ell$ under applied shear stress $\sigma_{x y}^{\text {ext }}$. We neglect the crystal structure and the Peierls potential [85]. Using the Peach-Koehler theory [86], we may calculate the elastic energy of the slip in isotropic elasticity as [70,

$$
F_{\text {slip }}=\frac{G b^{2}}{2 \pi} \frac{\ln (\ell / b)}{1-\nu} \mp \sigma_{x y}^{\text {ext }} b \ell \cos (2 \varphi),
$$

where - is for type $\mathrm{C},+$ is for type $\mathrm{CC}, b$ is the lattice constant, $G$ is the shear modulus, $\nu$ is Poisson's ratio, and $\varphi$ is the angle between the slip direction and the $x$ axis. The first term is the elastic energy of the dislocations, while the second term is the work of the applied force. For simple shear deformation with $\sigma_{x y}^{\text {ext }}>0$, the most favorable slip orientation with the lowest $F_{\text {slip }}$ is $\varphi=0$ (along the $x$ axis) for type $\mathrm{C}$ and $\varphi=\pi / 2$ (along the $y$ axis) for type CC. For such slips, the negativity $F_{\text {slip }}<0$ is eventually realized with increasing $\sigma_{x y}^{\text {ext }} \ell$, where the dislocations glide until they are pinned at defects. In the plastic flow regime, we should set

$$
\sigma_{x y}^{\mathrm{ext}} \sim G \bar{\gamma}_{\mathrm{el}} \sim \bar{\sigma}_{x y}
$$

where $\bar{\gamma}_{\text {el }}$ is introduced in Eq.(28). In real crystal, however, the critical stress causing dislocation motion can strongly depend also on the orientation of the glide plane with respect to the crystal axes [3]. As stated above, in our simulation, large-scale slip extensions tend to be induced when one of the crystal axes become nearly parallel to the $x$ or $y$ axis. Remarkably, we observed the same preferred directions in polycrystal and glass (see Fig.6 below). Namely, the grain boundary sliding is easily induced along the $x$ or $y$ axis (see Fig.13 also).

Experiments of plastic flow have been performed under uniaxial stress in metallurgy. The counterpart of Eq.(30) is given by [70]

$$
F_{\text {slip }}=\frac{G b^{2}}{2 \pi} \frac{\ln (\ell / b)}{1-\nu} \mp \sigma_{a}^{\text {ext }} \sin (2 \varphi),
$$

where $\sigma_{a}^{\text {ext }}=\left\langle\sigma_{x x}-\sigma_{y y}\right\rangle$ is the applied uniaxial stress. The most favorable direction is given by $\varphi=-\pi / 4$ for type $\mathrm{C}$ and $\varphi=\pi / 4$ for type CC. These preferred directions have been observed in amorphous metals [4, 64, and granular materials 34. In simulations, shear bands in these preffered directions have been realized in model amorphous metals and polymers 65 69 and in a model crystal with weak elastic anisotropy 70 . 


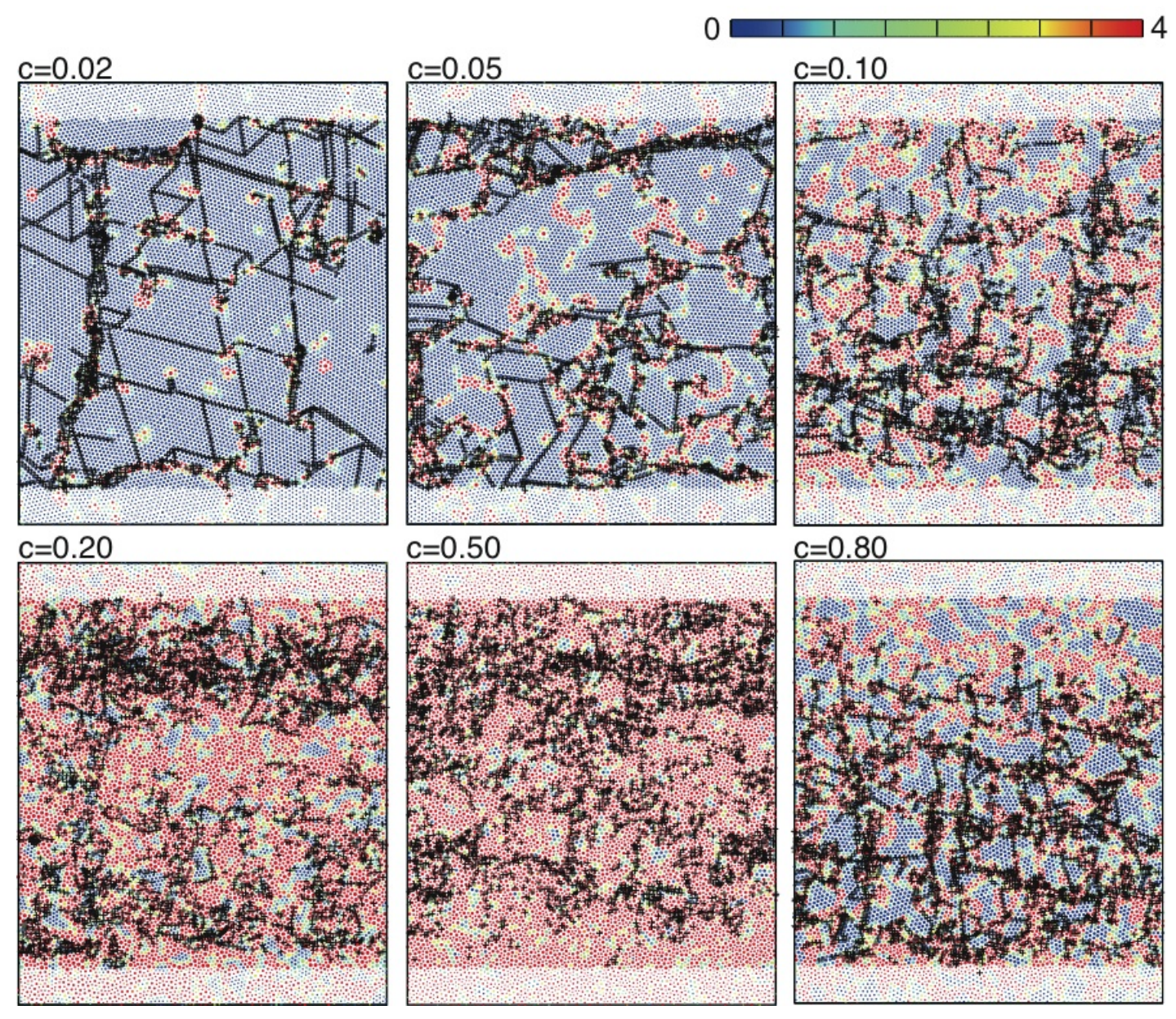

FIG. 6: (Color online) Broken bonds $(\times)$ in the time interval $\left[8 \times 10^{3}, 10^{4}\right]$ and disorder variable $D_{j}$ in Eq. 11 at the terminal time $t=10^{4}$ of the interval after application of shear $\dot{\gamma}=10^{-4}$, where $c=0.02,0.05,0.1,0.2,0.5$, and 0.8 in the six panels. Aggregates of broken bonds represent strain localization. The colors of the particles are given according to the color bar.

\section{F. Collective yielding and averaged velocity on long time scales $\left(\Delta t \geq 10^{3}\right)$}

First, on long time scales with $\Delta t \geq 10^{3}$, we demonstrate that plastic deformations occur collectively on large spatial scales, often on the system-size scale $L$ ( $\sim 100$ here $)$ at any $c$, while the type of deformations strongly depend on $c$.

Figure 6 shows the broken bonds in the time interval $\left[8 \times 10^{3}, 10^{4}\right]=[0.8 / \dot{\gamma}, 1 / \dot{\gamma}]$ and the disorder variable $D_{j}$ at the terminal time $t=10^{4}$ for $c=$ $0.02,0.05,0.1,0.2,0.5$, and 0.8 . Here a crystal state is realized for $c=0.02$, glass states for $c=0.2$ and 0.5 , and polycrystal states for the other cases. At the smallest $c=0.02$, most bond breakage processes occur in the form of extended dislocation gliding. A thickened grain boundary in the left part is produced by multiple sliding processes (see the left panel of Fig.7 also). For $c=0.05$, dislocation gliding occurs with shorter slip lengths. For $c=0.1$ and 0.8 , the bond breakage concentrates around the grain boundaries resulting in their sliding, in accord with Fig.5. For $c=0.2$ and 0.5 , the particle configurations are much disordered, but there is still a tendency of the particle motions along the boundaries of small crystalline regions, as was reported in our previous work [58. In all these panels taken for time intervals of $0.2 / \dot{\gamma}$, heterogeneities of broken bonds indicate emergence of large-scale shear bands with high strain localization. Furthermore, they mostly develop nearly along the $x$ or $y$ axis. At small $c$, the dislocation mechanism dominates and these features can be explained by the slip energy in Eq.(30). Remarkably, this orientation preference can be seen also in glass. With the boundary layers, the horizontal shear bands are more frequent than the vertical ones in our case, while using the Lees-Edwards boundary condition equally produced horizontal and vertical large-scale shear bands [27]. In an experiment on a Laponite suspension 26, yielding and the velocity field sensitively depended on whether the boundary wall is rough or smooth.

Figure 7 shows the broken bonds for $c=0.02$ in the longer time interval $\left[5 \times 10^{3}, 10^{4}\right]=[0.5 / \dot{\gamma}, 1 / \dot{\gamma}]$ and the orientation angles $\alpha_{j}$ defined in Eq. 10 . The left panel 


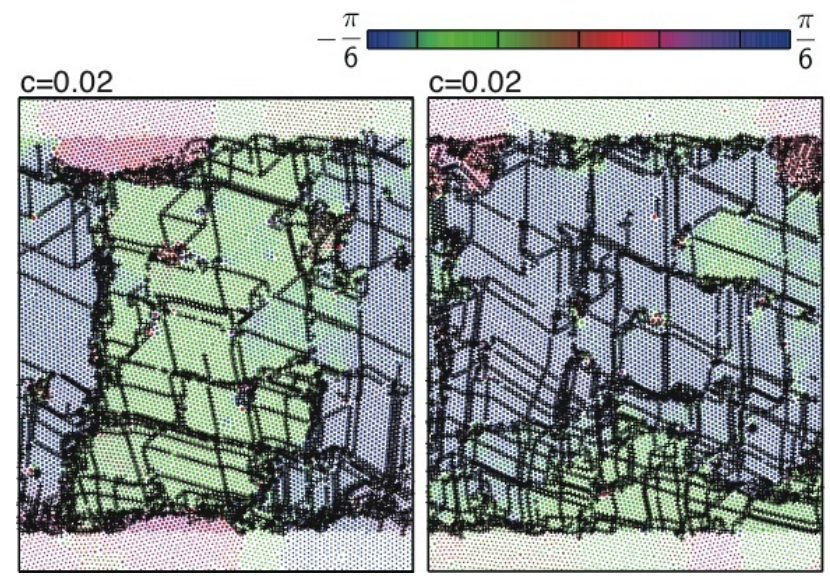

FIG. 7: (Color online) Broken bonds $(x)$ in the time interval $\left[5 \times 10^{3}, 10^{4}\right]$ and orientation angles $\alpha_{j}$ in Eq. 10 at the terminal time $t=10^{4}$ after application of shear for $c=0.02$. The colors are given according to the color bar. Left panel is obtained from the run yielding the left top panel in Fig.6. Right panel is obtained from an independent run, where the strain is localized in the lower part.
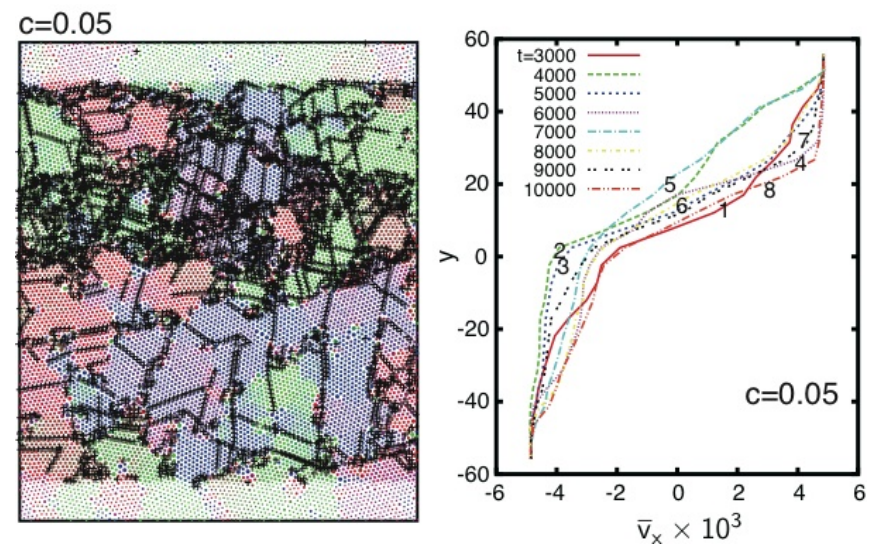

FIG. 8: (Color online) Left: Broken bonds in the time interval $\left[5 \times 10^{3}, 10^{4}\right]$ and orientation angles $\alpha_{j}$ at the terminal time $t=$ $10^{4}$ for $c=0.05$, where a long-lived shear band is developed. Right: Averaged velocity $\bar{v}_{x}(y, t)$ defined by Eqs.(33) and (34) at consecutive times $t=(2+n) \times 10^{3}(n=1, \cdots$, and 8) with $\Delta t=10^{3}$. Strain is localized in the upper part.

is the result from the run producing the left top panel of Fig.6, where the system is divided into two grains since the left and right regions are connected in our simulation. The difference in the orientation angles of the two grains is about $\pi / 12$. If we compare the left panel with the left top panel of Fig.6, we recognize accumulation of dislocation gliding and grain boundary sliding in the same narrow regions. The right panel of Fig. 7 is produced by an independent run, where we can see a thickened shear band composed of many broken bonds in the lower part.

In our system, shear bands disappear on long time scales as in Ref. 27, but they are sometimes observed to be long-lived. The left panel of Fig 8 shows such a tran-
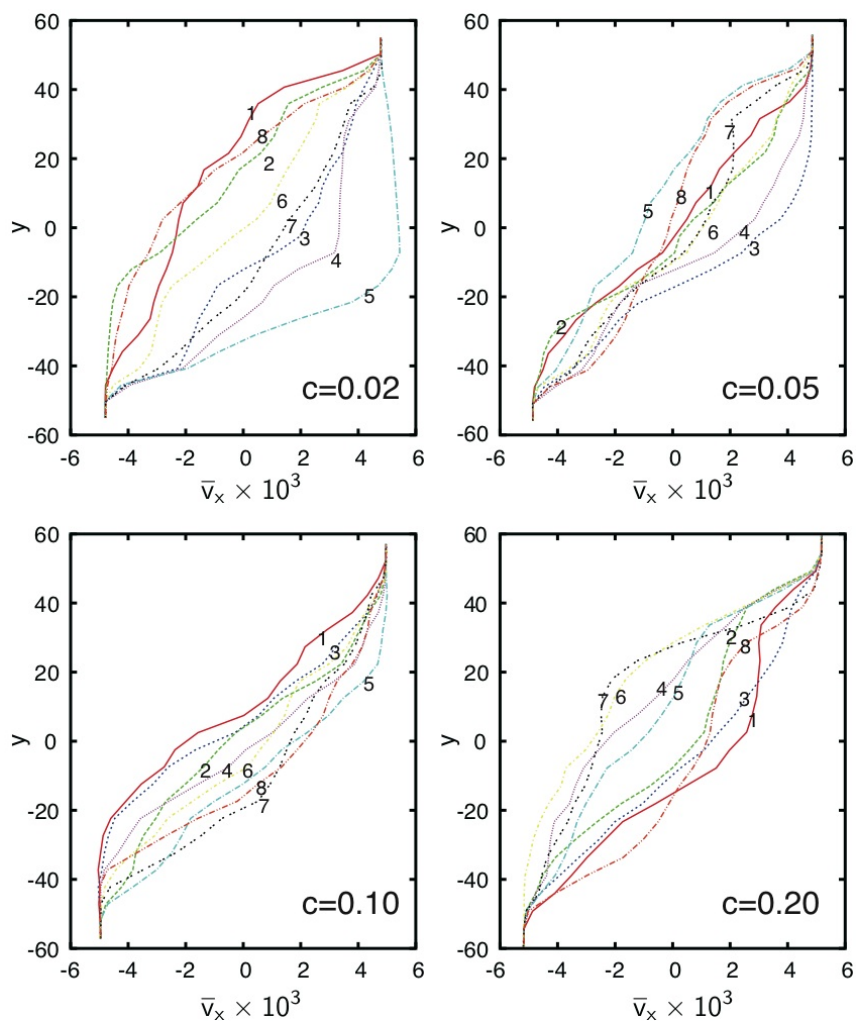

FIG. 9: (Color online) Consecutive proflies of averaged velocity $\bar{v}_{x}(y, t)$ defined by Eqs. (33) and (34) with $\Delta t=10^{3}=$ $0.1 / \dot{\gamma}$ for $c=0.02,0.05,0.10$, and 0.20 . Each curve is a result from a single run. Curves with label $n$ are made at $t=(2+n) \times 10^{3}$. Space-time fluctuations are conspicuous on this time scale of $10^{3}$ at any $c$.

sient shear band in the middle region for $c=0.05$, which was existent in the time region $2 \times 10^{3} \lesssim t \lesssim 1.6 \times 10^{4}$ but disappeared subsequently on a time scale of $10^{3}$. The right panel of Fig 8 gives profiles of an averaged velocity $\bar{v}_{x}(y, t)$ at different times in a single run. It exhibits large gradients along the $y$ axis in the band region with rather small temporal fluctuations.

To define $\bar{v}_{x}(y, t)$ in Fig. 8 , we first integrate the $x$ component of the momentum density $J_{x}(x, y, t)$ in the flow direction to obtain

$$
\begin{aligned}
\bar{J}_{x}(y, t) & =\frac{1}{L} \int_{-L / 2}^{L / 2} d x J_{x}(x, y, t) \\
& =\frac{1}{L} \sum_{j} m_{j} \dot{x}_{j}(t) \delta\left(y-y_{j}(t)\right) .
\end{aligned}
$$

We next average $\bar{J}_{x}(y, t)$ over space and time intervals with widths $\Delta y$ and $\Delta t$ to obtain

$$
\bar{v}_{x}(y, t)=\frac{1}{\bar{\rho} \Delta y \Delta t} \int_{y-\Delta y / 2}^{y+\Delta y / 2} d y^{\prime} \int_{t}^{t+\Delta t} d t^{\prime} \bar{J}_{x}\left(y^{\prime}, t^{\prime}\right)
$$

where $\bar{\rho}$ is the average mass density. We fix $\Delta y$ at $L / 20$. However, we set $\Delta t=10^{3}=0.1 / \dot{\gamma}$ in Figs. 8 and 9 , 

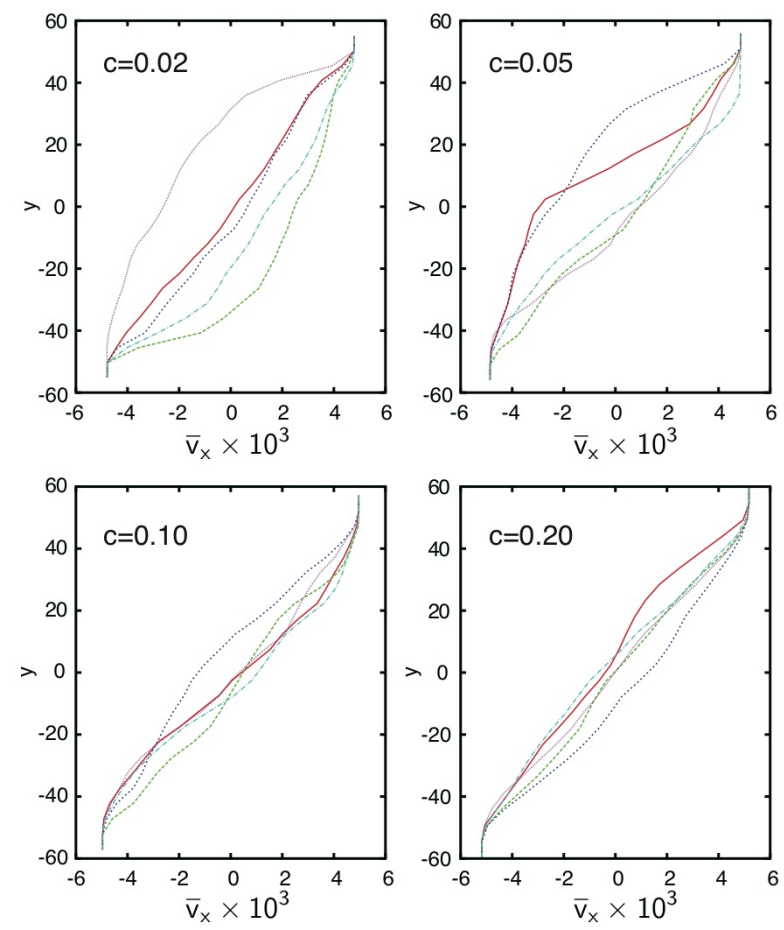

FIG. 10: (Color online) Averaged velocity $\bar{v}_{x}(y, t)$ with longer $\Delta t=8 \times 10^{3}=0.8 / \dot{\gamma}$ at $t=2 \times 10^{3}$ for $c=0.02,0.05,0.1$, and 0.2 . Five curves in each panel are obtained from independent runs. For $c=0.02$ and 0.05 , they much deviate from the linear profile depending on the initial conditions. For $c=0.1$ and 0.2 , they are nearly linear for any runs.

$\Delta t=8 \times 10^{3}$ in Fig.10, and $\Delta t=50$ in Figs.11 and 12 to analyze the hierarchical dynamics of plastic flow spanning these time scales.

Figure 9 shows eight consecutive profiles of the averaged velocity $\bar{v}_{x}(y, t)$ with $\Delta t=10^{3}=0.1 / \dot{\gamma}$ for $c=0.02,0.05,0.1$, and 0.2 in the four panels. For these runs, $\bar{v}_{x}(y, t)$ mostly deviates from the linear profile $\dot{\gamma} y$. Their space-time fluctuations are most enhanced at the smallest composition $c=0.02$. Visualized in these timeevolutions are emergence and movement of fragile areas on the time scale of $\Delta t=10^{3}$.

Figure 10 shows profiles of the averaged velocity $\bar{v}_{x}(y, t)$ with longer $\Delta t=8 \times 10^{3}=0.8 / \dot{\gamma}$ at $t=2 \times 10^{3}$ for $c=0.02,0.05,0.1$, and 0.2 . In each panel we write five curves obtained from independent runs. At $c=0.02$ and 0.05 , there still remain large deviations from the linear profile, sensitively dependent on the initial conditions of simulation. The curve in red solid line at $c=0.05$ is obtained from the run producing the shear band in Fig. 8. For such low compositions, the fragile areas are much extended and $\bar{v}_{x}(y, t)$ changes even on time scales longer than $1 / \dot{\gamma}$. For higher compositions at $c=0.1$ and 0.2 , the width $\Delta t=0.8 / \dot{\gamma}$ is sufficient to yield profiles close to the linear one. That is, with increasing $c$, large-scale plastic deformations become spatially uncorrelated if they are observed on time scales longer than $10^{4}=1 / \dot{\gamma}$.

\section{G. Large stress drops and collective plastic events on short time scales $(\Delta t \leq 50)$}

Figure 2 has shown that large stress drops can occur on a rapid time scale of $\tau_{\mathrm{ac}}$ in Eq.(21). It is of great interest how collective plastic deformations develop over large areas in short times, involving many particles.

We investigate this aspect for a polycrystal state with $c=0.05$ in Fig 11 and for a glass state with $c=0.2$ in Fig.12. In the upper panels of Figs.11 and 12, we present the shear stress $\left\langle\sigma_{x y}\right\rangle(t)$ in Eq.(14) and the histograms of the broken bond number $\Delta N_{b}(t)$ taken in time interval $[t-10, t]$ with width 10 . The lower panels display snapshots of the broken bonds and the disorder variable $D_{j}$ at $t=6890,7130$, and 7300 in Fig 11 and at $t=16730,17050$, and 17140 in Fig.12. No largescale yielding occurs in the first time interval [6840,6890] in Fig.11 and in the second time interval [17000, 17050] in Fig.12, where $\left\langle\sigma_{x y}\right\rangle(t)$ linearly grows and $\Delta N_{b}(t)$ remains small. In the other time intervals, correlated bondbreakge events are visualized in the forms of chains in Fig.11 and aggregates in Fig.12. They take place within the interval width 50 , inducing large $\Delta N_{b}(t)$ and abrupt drops of $\left\langle\sigma_{x y}\right\rangle(t)$. As in Figs.6-8, the large-scale plastic deformations tend to extend in directions nearly parallel to the $x$ or $y$ axis. These plastically deformed regions are inceptions of shear bands and are much longer than the grains in polycrystal and the small remaining crystalline regions in glass.

The averaged velocity $\bar{v}_{x}(y, t)$ defined in Eqs. (33) and (34) are also calculated in Figs.11 and 12, where the smoothing time interval $\Delta t=50$ is much shorter than in Figs.9 and 10. For $c=0.05$ in Fig.11, $\bar{v}_{x}(y, t)$ largely deviates from the linear profile even without collective yielding at $t=6890$. This suggests that short-time deformations can be nonaffine even with weak bond breakage, which is in accord with the simulations in Ref. 60, 61. In the third time interval, the upper part contains largescale plastic deformations and move in the $x$ direction ahead of the upper boundary, while the lower part moves back in the negative $x$ direction. Also for $c=0.2$ in Fig. $12, \bar{v}_{x}(y, t)$ much deviates from the linear profile in the presence of collective yielding, while it happens to be rather close to the linear profile in the second interval without collective yielding.

In Fig. 13, we display the particle displacement,

$$
\Delta \boldsymbol{r}_{j}(t)=\boldsymbol{r}_{j}(t)-\boldsymbol{r}_{j}(t-\Delta t)
$$

with $\Delta t=50$ around chains or aggregates of the broken bonds, expanding active regions of yielding with area $L^{2} / 16$ in Figs.11 and 12 . We pick up the particles with displacement $\left|\Delta \boldsymbol{r}_{j}(t)\right|$ longer than $0.3 \sigma_{1}$ suppressing those with smaller displacements. The number of such "mobile" particles is much larger than the number of the broken bonds in the same time interval. In Table 1, the broken bond number is about 200 (about 30 in the narrow regions in Fig.13), while the number of the mobile particles is from a few to several thousands. 


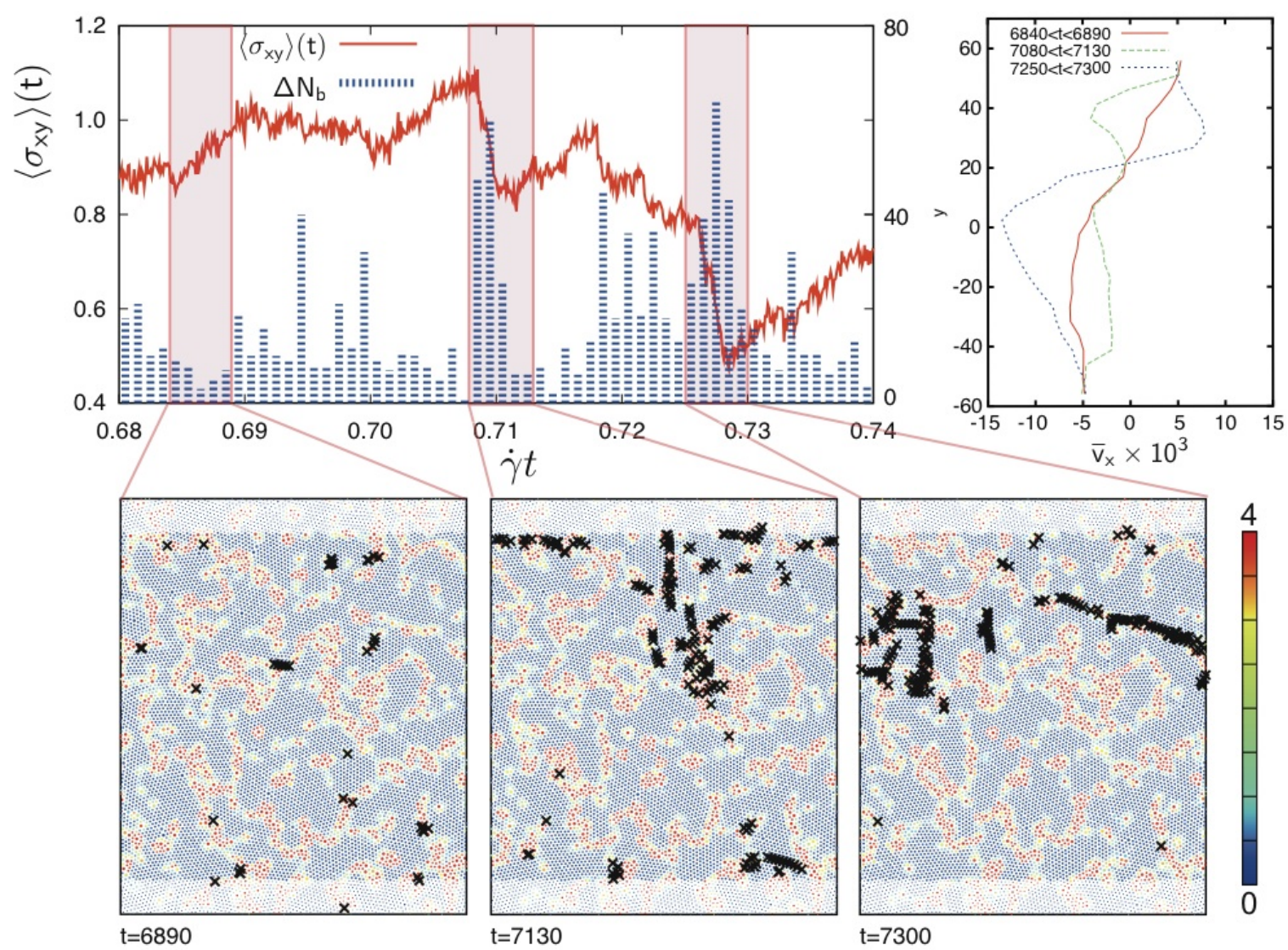

FIG. 11: (Color online) Left top: Shear stress $\left\langle\sigma_{x y}\right\rangle(t)$ (red bold line with left scale) and broken bond number $\Delta N_{b}(t)$ in time interval with width 10 (blue broken bars with right scale) as functions of strain $\dot{\gamma} t$ in polycrystal with $c=0.05$. Bottom: Broken bonds at $t=6890,7130$, and 7300 in time interval with width 50 and disordered variable $D_{j}$ at these times. In the first time interval [6840,6890], there is no large-scale plastic deformation, where $\left\langle\sigma_{x y}\right\rangle(t)$ linearly grows and $\Delta N_{b}(t)$ are small. In the second and third time intervals $[7080,7130]$ and $[7250,7300],\left\langle\sigma_{x y}\right\rangle(t)$ drops and $\Delta N_{b}(t)$ are large. Right top: Averaged velocity $\bar{v}_{x}(y, t)$ at these times with $\Delta t=50$.

The differences of these numbers are because the bond breakage picks up the particle motions most significantly contributing to plastic deformations. The broken bonds form long chains for $c=0.05$ and aggregates of strings for $c=0.2$ along the $y$ axis (left panels) and along the $x$ axis (right panels). The displacement vectors tend to be clockwise around horizontal chains or aggregates of the broken bonds and counterclockwise around vertical ones. In Fig.14, by setting $\Delta t=10$, we furthermore show that short slip lines in glass successively appear in their neigborhood for $c=0.2$.

We then analyze how many particles are involved at large stress drops. To this end, we rewrite the shear strss in Eq.(14) in the sum $\left\langle\sigma_{x y}\right\rangle(t)=\sum_{j} \mathcal{S}_{j}(t) / L^{2}$ with

$$
\mathcal{S}_{j}(t)=m_{j} \dot{x}_{j} \dot{y}_{j}-\sum_{k} \frac{x_{j k} y_{j k}}{2 r_{j k}} \phi_{j k}^{\prime}
$$

Since the stress drop in the time interval $[t-\Delta t, t]$ is written as $\sum_{j}\left[\mathcal{S}_{j}(t)-\mathcal{S}_{j}(t-\Delta t)\right] / L^{2}$, we may introduce the particle number distribution defined by

$$
P(w)=\sum_{j} \delta\left(\mathcal{S}_{j}(t)-\mathcal{S}_{j}(t-\Delta t)-w\right) .
$$

Then $\int d w P(w)=N$ and

$$
\int d w w P(w)=L^{2}\left[\left\langle\sigma_{x y}\right\rangle(t)-\left\langle\sigma_{x y}\right\rangle(t-\Delta t)\right]
$$

is equal to the stress drop multiplied by $L^{2}$. In Fig.15, we give histograms of $w P(w)$ in regions $n-1 / 2<w / \epsilon<n+$ $1 / 2(n=0, \pm 1, \pm 2, \cdots)$ in the time interval $[7250,7280]$ for $c=0.05$ and in the time interval $[17090,17140]$ for $c=0.2$, where the shear stress largely drops in Figs.11 and 12. Broad distrubutions of $w$ are due to the thermal fluctuations at high pressure, but asymmetry between the negative and positive regions of $w$ can be seen around $w \sim \pm 5 \epsilon$. As a result, the integral $\int d w w P(w)$ is equal to $-3.15 \times 10^{3}$ for $c=0.05$ and to $-1.19 \times 10^{3}$ for $c=0.2$. 


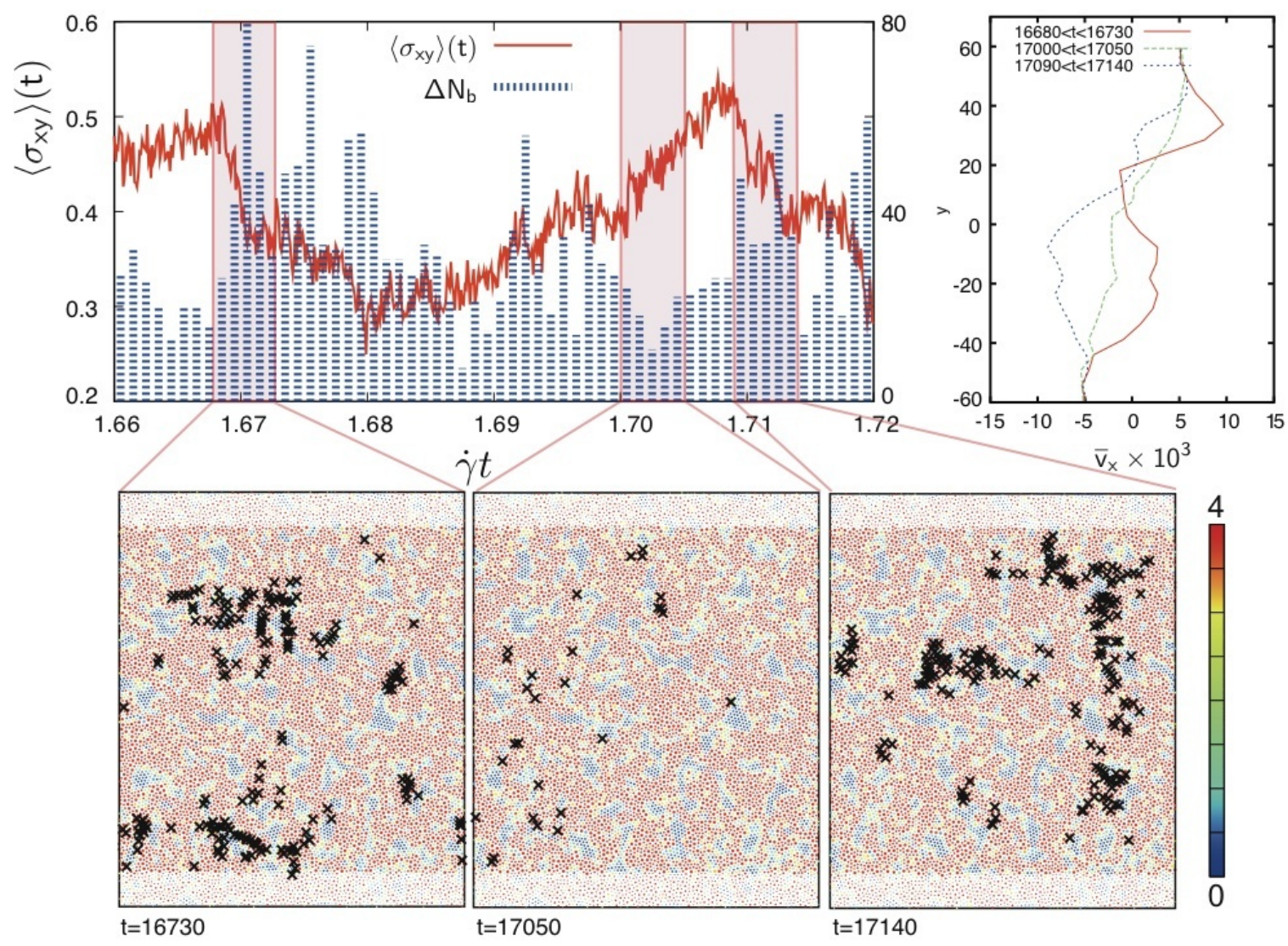

FIG. 12: (Color online) Top left: Shear stress $\left\langle\sigma_{x y}\right\rangle(t)$ (red solid line with left scale) and broken bond number $\Delta N_{b}(t)$ in time interval with width 10 (blue broken bars with right scales) as functions of strain $\dot{\gamma} t$ in glass with $c=0.2$. Bottom: Broken bonds at $t=16730,17050$, and 17140 in time interval with width 50 and disordered variable $D_{j}$ at these times. Large-scale plastic deformations do not occur in the second time interval $[17000,17050]$ but occur in the first and third time intervals $[16680,16730]$ and $[17090,17140]$. Right top: Averaged velocity $\bar{v}_{x}(y, t)$ at these times with $\Delta t=50$.

TABLE I: Numbers of broken bonds and mobile (largely displaced) particles in the total bulk region at four time intervals with width 50 in Fig.13. Stress drop $\left\langle\bar{\sigma}_{x y}\right\rangle(t)-\left\langle\bar{\sigma}_{x y}\right\rangle(t-50)$ (bottom) is large.

\begin{tabular}{ccccc} 
& $(\mathrm{a})$ & $(\mathrm{b})$ & $(\mathrm{c})$ & $(\mathrm{d})$ \\
\hline$c$ & 0.05 & 0.05 & 0.2 & 0.2 \\
\hline broken bonds & 170 & 180 & 180 & 200 \\
\hline mobile particles & 1820 & 5200 & 3500 & 3100 \\
\hline stress drop & -0.168 & -0.278 & -0.100 & -0.133 \\
\hline
\end{tabular}

\section{SUMMARY AND REMARKS}

Though in two dimensions, we have treated crystal, polycrystal, and glass in a unified manner by varying the composition $c$ with $\sigma_{2} / \sigma_{1}=1.4$ and $T=0.2 \epsilon / k_{B}$ held fixed. Shear flow with $\dot{\gamma}=10^{-4}$ has been realized with Nosè-Hoover thermostats attached to the top and bottom boundary layers. We summarize our main results.

(i) In Sec.IIIC, we have calculated the spatially averaged stress $\left\langle\sigma_{x y}\right\rangle(t)$ in Eq.(14) and the temporally smoothed wall stress $\bar{\sigma}_{w}(t, \Delta t)$ in Eq.(20) as in Fig.1, which tend to coincide with increasing the smoothing time interval $\Delta t$. The former has been calculated in the literature but is not directly observable, while the latter is observable. (ii) In Sec.IIID, $\left\langle\sigma_{x y}\right\rangle(t)$ has been displayed together with the histograms of the broken bond number $\Delta N_{b}(t)$ in Fig.2. Here stress drops are related to increases in the bond breakage. Also the plastic strain increment $\Delta \gamma_{\mathrm{pl}}$ and the elastic strain increment $\Delta \gamma_{\mathrm{el}}$ have been phenomenologically introduced in Eqs.(25) and (27).

(iii) In Sec.IIIE, the dislocation gliding and the grain boundary sliding have been studied in Figs.4 and 5. From the elastic energy of a slip under applied stress in Eq.(30), we have argued why the plastic deformations tend to be nearly along the $x$ or $y$ axis and why the particle displacement vectors are clockwise (type C) around slips along the $x$ axis and counterclockwise (type CC) around slips along the $y$ axis. Though these are arguments for slips, we have found the same preferred directions and displacements around broken bonds for any $c$.

(iv) In Sec.IIIF, visualization of the dynamic and structural heterogeneities have been given for various $c$. Displayed is the bond breakage in the background of the disorder variable $D_{j}$ with $\Delta t=0.2 / \dot{\gamma}$ in Fig. 6 and in the 


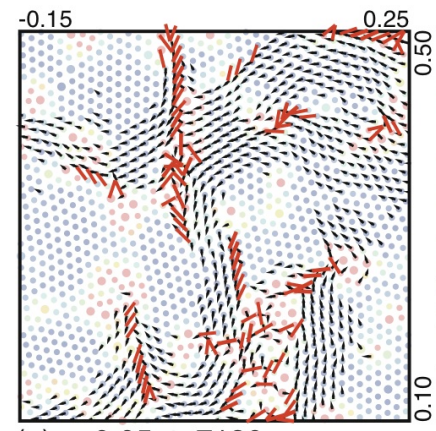

(a) $\mathrm{c}=0.05, \mathrm{t}=7130$

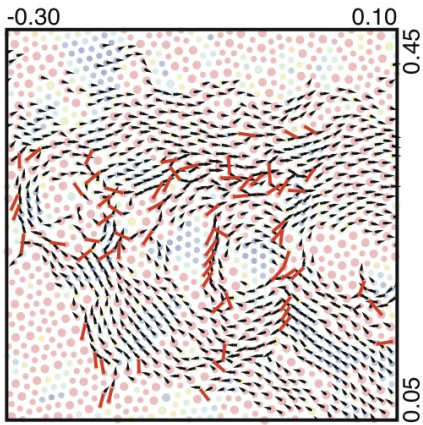

(c) $\mathrm{c}=0.20, \mathrm{t}=16730$

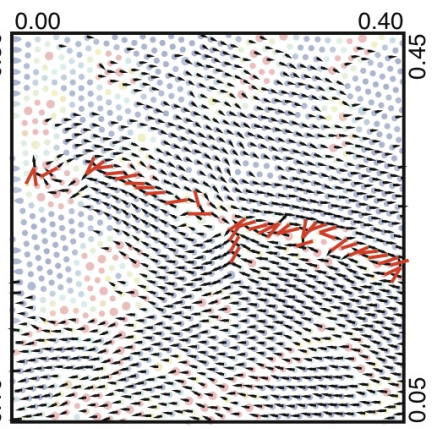

(b) $\mathrm{c}=0.05, \mathrm{t}=7300$

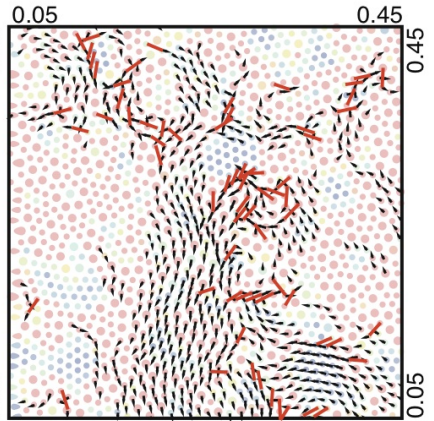

(d) $\mathrm{c}=0.20, \mathrm{t}=17140$

FIG. 13: (Color online) Broken bonds (red solid lines) and particle displacements with $\left|\boldsymbol{r}_{j}(t)-\boldsymbol{r}_{j}(t-50)\right|>0.3 \sigma_{1}$ (arrows) in a time interval $[t-50, t]$ obtained from the runs producing Figs.11 and 12, where (a) $c=0.05$ and $t=7130$, (b) $c=0.05$ and $t=7300$, (c) $c=0.2$ and $t=16730$, and (d) $c=0.2$ and $t=17140$. The arrow are from the initial to final positions. In (a) and (b) broken bonds are along the grain boundaries. For (c) and (d) time-evolution of broken bonds is given in Fig.14. Parts of $16 \%$ of the total bulk region are displayed.

(c) $c=0.20$

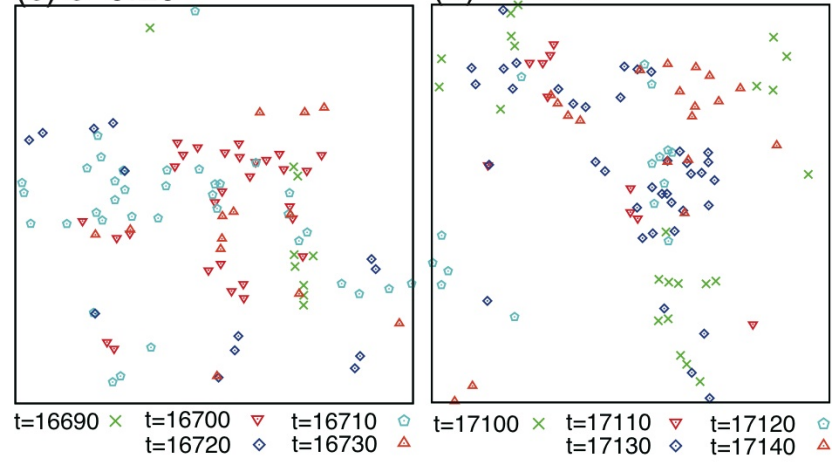

FIG. 14: (Color online) Broken bonds in five consecutive time intervals $[t-10, t]$ with width $\Delta t=10$ for $c=0.2$, where $t=16730-10 n$ (left) and $t=17140-10 n$ (right) with $0 \leq n \leq 4$, corresponding to the lower panels (c) and (d) of Fig.13.
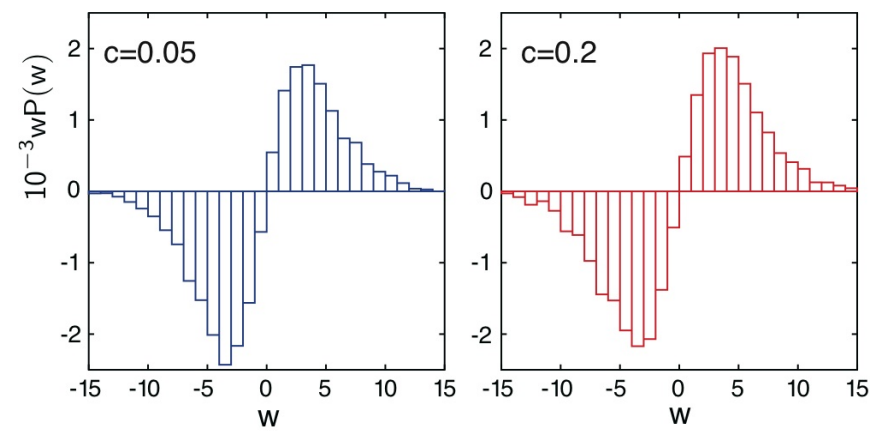

FIG. 15: (Color online) Histograms of $w P(w)$ defined by Eqs.(37) and (38) with $w$ in units of $\epsilon$ in the time interval [7250,7280] for $c=0.05$ in Fig.11 (left) and the time interval [17090, 17140] for $c=0.2$ in Fig.12 (right).

background of the orientation angles $\alpha_{j}$ with $\Delta t=0.5 / \dot{\gamma}$ in Figs.7 and 8. The overall heterogeneity directions are along the $x$ or $y$ axis for any $c$. The averaged velocity $\bar{v}_{x}(y, t)$ defined by Eqs.(33) and (34) represents the mean particle motions along the $x$ axis. For $\Delta t=0.1 / \dot{\gamma}$ in Fig.9, it greatly deviates from the linear profile for any c. For $\Delta t=0.8 / \dot{\gamma}$ in Fig.10, its deviation still remains for small $c$ but becomes small for not small $c$.

(v) In Sec.IIIG, catastrophic plastic events at large stress drops have been visualized in Figs.11-15 for $c=0.05$ (polycrystal) and 0.2 (glass) with $10 \leq \Delta \leq 50$. In Fig.14, time-evolution of broken bonds in sheared glass shows how they appear as short slips and how they aggregate on a time scale of $\Delta t=10$.

We give concluding remarks in the following.

(1) For any $c$, plastic events extend over a mesoscopic area $\left(\gg \sigma_{1}\right)$ and occur in a short time, as visualized on a time scale of $\Delta t=50$ in Figs.11 and 12. In crystal and polycrystal plasticity is due to slip formation and grain boundary sliding with various sizes $[4,5$. In glass, slip lines are short but collectively appear in a narrow region, as visualized in Fig.14. Subsequent plastic events tend to multiply occur in such fragile areas to form shear bands, which can extend thoughout the system for our (still small) system size on much longer time scales of order $10^{3}$ as in Figs.6-9. On time scales longer than $10^{4}=1 / \dot{\gamma}$, the correlations of plastic events to earlier ones still persist for small $c$ but disappear in glass as in Fig.10. This long-range cooperativity propagates in short times and persist for long times decaying slower in polycrystal than in glass.

2) Molecular glassy materials behave as elastic bodies on short time scales, though mesoscopically inhomogeneous elastic moduli give rise to irregular deformations [60, 61. Large-scale intermittent release of the elastic energy at high stress is a common feature of plasticity in crystal, polycystal, and glass. See Figs.5 and 14, where nonlinear deformations propagate rapidly as long or short slips depending on $c$.

(3) We remark on the dynamic heterogeneity in glass without shear [48 59. Jammed particle configuration 
changes are first triggered in the form of chains of broken bonds [17, 51] or stringlike clusters [52, 54] in microscopic times, which are mostly around boundaries of small remaining crystalline regions [58, 59]. Subsequently, the clusters of the broken bonds accumulate to form mesoscopic heterogeneities on long time scales. To confirm this process, some papers have visualized time-evolution of the dynamic heterogeneites [17, 51, 55, 71, where succesive broken bonds or active regions mostly overlap or are adjacent to each other. Applied shear intensifies this tendency, eventually leading to organization of shear bands, as illustrated in this paper.

(4) In the melting phenomena in two dimensions, we remark that analogous large-scale heterogeneities emerge both in the structural disorder and in the dynamics [76].

\section{Acknowledgments}

The authors are grateful to T. Hamanaka, T. Araki, T. Uneyama, S. Yukawa, and A. Furukawa for valuable discussions. Some of the numerical calculations were carried out on NEC SX8 at YITP in Kyoto University. This work was supported by Grants-in-Aid for scientific research on Priority Area "Soft Matter Physics" and the Global COE program "The Next Generation of Physics, Spun from Universality and Emergence" of Kyoto University from the Ministry of Education, Culture, Sports, Science and Technology of Japan. H. S. was supported by the Japan Society for Promotion of Science.

\section{Appendix: Microscopic Expressions for Aver- age Stress and Forces to Walls}

Our system is composed of the particles unbound and bound to the top and bottom walls in the regions $-0.6 L<y<-0.5 L$ and $0.5 L<y<0.6 L$. The total potential energy of our system is written as Eq.(5). From the equations of motion we derive the following equation,

$\frac{d}{d t} \sum_{j \in \text { all }} m_{j} y_{j} \dot{x}_{j}=\Pi_{x y}^{0}-\sum_{\mathcal{B}} \sum_{j \in \mathcal{B}}\left[y_{j} \frac{\partial u_{j}}{\partial x_{j}}+\zeta_{\mathcal{B}} m_{j} y_{j}\left(\dot{x}_{j}-v_{\mathcal{B}}\right)\right]$, where $\ddot{x}_{j}=d^{2} x_{j} / d t^{2}, \dot{x}_{j}=d x_{j} / d t, \partial u_{j} / \partial x_{j}=K\left[x_{j}-\right.$ $\left.X_{j}(t)\right]$, and $v_{\mathcal{B}}= \pm \dot{\gamma} L / 2$. The subscript $\mathcal{B}$ denotes the top or bottom boundary. We introduce the space integral of the $x y$ component of the stress of all the particles,

$$
\Pi_{x y}^{0}=\sum_{j \in \text { all }} m_{j} \dot{x}_{j} \dot{y}_{j}-\sum_{j, k \in \text { all }} \frac{x_{j k} y_{j k}}{2 r_{j k}} \phi_{j k}^{\prime}
$$

where $\dot{y}_{j}=d y_{j} / d t, r_{j k}=\left|\boldsymbol{r}_{j}-\boldsymbol{r}_{k}\right|$, and $\phi_{j k}^{\prime}=\partial \phi_{j k} / \partial r_{j k}$.

The total forces acted by the walls to the fluid along the $x$ axis are given by $F_{\text {top }}$ and $F_{\text {bot }}$ in Eq. (15). From Eqs.(A1) and (A2) the average stress $\left\langle\sigma_{x y}\right\rangle(t)$ in Eq. (14) and the wall stress $\sigma_{\mathrm{w}}(t)$ in Eq. (13) are related as

$$
\begin{aligned}
& L^{2}\left[\sigma_{\mathrm{w}}(t)-\left\langle\sigma_{x y}\right\rangle(t)\right]=\frac{d}{d t} \sum_{j \in \text { all }} m_{j} y_{j} \dot{x}_{j}-\Delta \Pi_{x y}^{0} \\
& +\sum_{\mathcal{B}} \sum_{j \in \mathcal{B}}\left(y_{j} \mp \frac{L}{2}\right) \frac{\partial u_{j}}{\partial x_{j}}+\sum_{\mathcal{B}} \sum_{j \in \mathcal{B}} \zeta_{\mathcal{B}} m_{j} y_{j}\left(\dot{x}_{j}-v_{\mathcal{B}}\right) .
\end{aligned}
$$

where - is for the top layer and + is for the bottom layer in the second line. Here we write the contribution to $\Pi_{x y}^{0}$ from the bound particles as

$$
\Delta \Pi_{x y}^{0}=\sum_{\mathcal{B}}\left[\sum_{j \in \mathcal{B}} m_{j} \dot{x}_{j} \dot{y}_{j}-\sum_{j \text { or } k \in \mathcal{B}} \frac{x_{j k} y_{j k}}{2 r_{j k}} \phi_{j k}^{\prime} .\right]
$$

In the right hand side of Eq.(A3), the first term is the time derivative of the sum $\sum_{j \in \text { all }} m_{j} y_{j} \dot{x}_{j}$ of all the particles, while the other terms involve the bound particles. Thus the first term is dominant in the limit of large $L$. This is indeed the case in our simulation. That is, in the right hand side, the third term is less than $10 \%$ of the first term and the second and fourth terms are much smaller than the third at any time.
[1] F. Spaepen, in Physics of Defects, edited by R. Balian, M. Kléman and J -P. Poirier, 1981 Les Houches Lectures (North-Holland, Amsterdam, 1981), p. 133.

[2] A.S. Argon, in Physical Metallurgy, edited by R.W. Cahn and P. Haasen (North-Holland, Amsterdam, 1996), p.1878, 1958; P. Haasen, ibid., p.2010; J.L. Strudel, ibid., p. 2106 .

[3] J. Friedel, Dislocations (Pergamon, New York, 1954).

[4] M.-C. Miguel, A. Vespignani, S. Zapperi, J. Weiss, and Jean-Robert Grassok, Nature 410, 667 (2001); T. Richeton, J. Weiss, and F. Louchet, Nature Materials 4, 465 (2005); M. Zaiser, Adv. Phys. 55, 185 (2006).
[5] D. M. Dimiduk, C. Woodward, R. LeSar, and M. D. Uchic, Science 312, 1188 (2006).

[6] B. Devincre and L. P. Kubin Mater. Sci. Eng. A 234236, 8 (1997); V. Bulatov, F.F. Abraham, L. Kubin, B. Devincre and S. Yip, Nature 391, 669 (1998).

[7] T. M. Pollock and A. S. Argon, Acta Mater. 40, 1 (1992).

[8] A. Minami and A. Onuki, Phys. Rev. B 70, 184114 (2004); Acta Mater. 55, 2375 (2007).

[9] H. Hahn, P. Mondal, and K. A. Padmanabhan, Nanostruct. Mater. 9, 603 (1997).

[10] J. Schiotz, F.D. Di Tolla, and K.W. Jacobsen, Nature 391,561 (1998). 
[11] A. Hasnaoui, H. Van Swygenhoven, and P.M. Derlet, Phys. Rev. B 66 , 184112 (2002).

[12] S. Yip, Nature Mater. 3 ,11 (2004).

[13] V. Yamakov, D. Wolf, S. R. Phillpot, A. K. Mukherjee, and H. Gleiter, Nature Materials 3 , 43 (2004).

[14] T. Hamanaka, H. Shiba, and A. Onuki, Phys. Rev. E 77, 042501 (2008).

[15] H. Zhang, D. J. Srolovitz, J. F. Douglas, and J. A. Warren, Phys. Rev. B 74, 115404 (2006); Proc. Nat. Acad. Sci. USA 106, 7735 (2009).

[16] J.H. Simmons, R.K. Mohr, and C.J. Montrose, J. Appl. Phys. 53, 4075 (1982). To be precise, they measured the elongational viscosity at constant elongation rate, which is written as $\dot{\gamma}$ in Sec.I.

[17] R. Yamamoto and A. Onuki, Europhys. Lett. 40, 61 (1997); Phys. Rev. E 58, 3515 (1998); J. Phys.: Condens. Matter 29, 6323 (2000).

[18] L. Angelani, G. Ruocco, F. Sciortino, P. Tartaglia, and F. Zamponi, Phys. Rev. E 66, 061505 (2002).

[19] M. Fuchs and M. E. Cates, Phys. Rev. Lett. 89, 248304 (2002); K. Miyazaki, D.R. Reichman, Phys. Rev. E 66, 050501(R) (2002).

[20] F. Varnik, L. Bocquet, J.-L. Barrat, and L. Berthier, Phys. Rev. Lett. 90, 095702 (2003).

[21] L. Berthier, J. Phys. Condens. Matter 15, S933 (2003).

[22] D.J. Lacks and M.J. Osborne, Phys. Rev. Lett. 93, 255501 (2004).

[23] A. Tanguy, F. Leonforte, and J.-L. Barrat, Eur. Phys. J. E 20, 355 (2006).

[24] C. E. Maloney and A. Lemaitre, Phys. Rev. E 74, 016118 (2006). A. Lemaitre and C. Caroli, Phys. Rev. E 76, 036104 (2007).

[25] Y. Shi, M. B. Katz, H. Li, and M. L. Falk, Phys. Rev. Lett. 98, 185505 (2007).

[26] T. Gibaud, C. Barentin, and S. Manneville, Phys. Rev. Lett. 101, 258302 (2008).

[27] A. Furukawa, K. Kim, S. Saito, and H. Tanaka, Phys. Rev. Lett. 102, 016001 (2009).

[28] N. P. Bailey, J. Schi $\phi$ tz, A. Lemaitre, and K. W. Jacobsen, Phys. Rev. Lett. 98, 095501 (2007); A. Lemaitre and C. Caroli, Phys. Rev. Lett. 103, 065501 (2009); H. G. E. Hentschel, S. Karmakar, E. Lerner, and I. Procaccia, Phys. Rev. Lett. 104, 025501 (2010).

[29] T. Okuzono and K. Kawasaki, Phys. Rev. E 51, 1246 (1995).

[30] D.J. Durian, Phys. Rev. E 55, 1739 (1997); S.A. Langer and A.J. Liu, J. Phys. Chem. B 101, 8667 (1997).

[31] P. Hébraud, F. Lequeux, J.P. Munch and D.J. Pine, Phys. Rev. Lett. 78, 4657 (1997).

[32] R. Besseling, E. R. Weeks, A. B. Schofield, and W. C. K. Poon, Phys. Rev. Lett. 99, 028301 (2007).

[33] P. Schall, D. A. Weitz, and F. Spaepen, Science 318, 1895 (2007).

[34] J. Desrues, J. Lanier and P. Stutz, Eng. Fra. Mech. 21, 909 (1985); J. Desrues and R. Chambon, Int.J. Solids Struct. 39, 3757 (2002).

[35] B. Miller, C.O'Hern and R.P. Behringer, Phys. Rev. Lett. 77, 3110 (1996).

[36] O.J. Schwarz, Y. Horie, and M. Shearer, Phys. Rev. E $\mathbf{5 7}, 2053$ (1998).

[37] O. Dauchot, G. Marty, and G. Biroli, Phys. Rev. Lett. 95, 265701 (2005).

[38] A. Fall, F. Bertrand, G. Ovarlez, and D. Bonn, Phys. Rev. Lett. 103, 178301 (2009).
[39] P. Olsson and S. Teitel, Phys. Rev. Lett. 99, 178001 (2007); T. Hatano, Phys. Rev. E 79, 050301(R) (2009).

[40] K. Binder and W. Kob, Glassy Materials and Disordered Solids (World Scientific, Singapore, 2005).

[41] F. Spaepen, Acta Metall. 25, 407 (1977).

[42] A.S. Argon, Acta Metall. 27, 47 (1979).

[43] M. L. Manning, J. S. Langer, and J. M. Carlson, Phys. Rev. E 76, 056106 (2007).

[44] C. A. Schuh, T. C. Hufnagel, and U. Ramamurty, Acta Materialia 55, 4067 (2007).

[45] H.S. Chen and M. Goldstein, J. Appl. Phys. 43, 1642 (1971).

[46] D. Srolovitz, V. Vitek, and T. Egami, Acta Metall. 31, 335 (1983).

[47] M. Zink, K. Samwer, W. L. Johnson, and S. G. Mayr, Phys. Rev. B 73, 172203 (2006).

[48] K. Maeda and S. Takeuchi, Phys. Stat. Sol. 49, 685 (1978); S. Kobayashi, K. K. Maeda and S. Takeuchi, Acta Metall. 28, 1641 (1980).

[49] T. Muranaka and Y. Hiwatari, Phys. Rev. E 51, R2735 (1995).

[50] M. M. Hurley and P. Harrowell, Phys. Rev. E 52, 1694 (1995); D.N. Perera and P. Harrowell, ibid. 54, 1652 (1996).

[51] R. Yamamoto and A. Onuki, J. Phys. Soc. Jpn., 662545 (1997).

[52] W. Kob, C. Donati, S. J. Plimpton, P. H. Poole, and S. C. Glotzer, Phys. Rev. Lett. 79, 2827 (1997).

[53] C. Donati, J. F. Douglas, W. Kob, S. J. Plimpton, P. H. Poole, and S. C. Glotzer, Phys. Rev. Lett. 80, 2338 (1998).

[54] N. Lačević, F. W. Starr, T. B. Schroder, and S. C. Glotzer, J. Chem. Phys. 119, 7372 (2003).

[55] B. Doliwa and A. Heuer, J. Non-Cryst. Solids 307-310, 32 (2002).

[56] G. Biroli, J.-P. Bouchaud, K. Miyazaki, and D.R. Reichman, Phys. Rev. Lett. 97, 195701 (2006).

[57] A. Widmer-Cooper and P. Harrowell, Phys. Rev. Lett. 96 (2006), 185701.

[58] T. Hamanaka and A. Onuki, Phys. Rev. E 74, 011506 (2006); ibid. 75, 041503 (2007).

[59] T. Kawasaki, T. Araki, and H. Tanaka, Phys. Rev. Lett. 99, 215701 (2007).

[60] K. Yoshimoto, T. S. Jain, K. Van Workum, P. F. Nealey, and J. J. de Pablo, Phys. Rev. Lett. 93, 175501 (2004).

[61] M. Tsamados, A. Tanguy, C. Goldenberg, and J.-L. Barrat, Phys. Rev. E 80, 026112 (2009).

[62] In this paper,we do not consider fracture or brittle behavior. We use the term "shear band" widely to represent quasi-linear (in 2D) mesoscopic objects where shear strains are localized.

[63] Jing Li, F. Spaepen and T.C. Hufnagel, Phil. Mag. A 82, 2623 (2002).

[64] Q. Wei, D. Jia, K. T. Ramesh, and E. Ma Appl. Phys. Lett. 81, 1240 (2002).

[65] D. Deng, A.S. Argon, and S. Yip, Phil. Trans. R. Soc. Lond. A 329, 613 (1989).

[66] Y. Shi and M. L. Falk, Phys. Rev. B 73, 214201 (2006).

[67] C. E. Maloney and M. O. Robbins, J. Phys.: Condens. Matter 20, 244128 (2008).

[68] N. P. Bailey, J. Schi $\phi$ tz, and K. W. Jacobsen, Phys. Rev. B 73, 064108 (2006).

[69] V.V. Bulatov and A.S. Argon, Modell. Simul. Mater. Sci. Eng. 2, 167 (1994); A.S. Argon, V.V. Bulatov, P.H. Mott 
and U.W. Suter, J. Rheol. 39, 377 (1995).

[70] A. Onuki, Phys. Rev. E 68, 061502 (2003); A. Onuki, A. Furukawa, and A. Minami, Pramana 64, 661 (2005).

[71] H. Shiba and A. Onuki, Prog. Theor. Phys. Suppl. (to be published).

[72] The minimums of the LJ potentials in Eq.(1) are at $r=$ $2^{1 / 6} \sigma_{\alpha \beta}$, so another nearly equivalent definition of the volume fraction is $\phi=D\left(N_{1} \sigma_{1}^{2}+N_{2} \sigma_{2}^{2}\right) / N$, where $D=$ $2^{1 / 3} \pi / 4 \cong 0.9895$ is close to unity.

[73] S. Nosè, Molec. Phys. 52, 255 (1983).

[74] W.G. Hoover, Computational Statistical Mechanics, (Elsevier, Amsterdam,1991).

[75] To be precise, for $\dot{\gamma}=10^{-4}$, the temperature in the middle region became higher than the boundary value 0.2 by a few $\%$ 71. We also performed simulations with $N=81000$, where the temperature inhomogeneity increased considerably at high shear with $\dot{\gamma} \gtrsim 10^{-3}$.

[76] H. Shiba, A. Onuki, and T. Araki, EPL 86, 66004 (2009).

[77] B.I. Halperin and D. R. Nelson, Phys. Rev. Lett. 41, 121 (1978).

[78] R. Zwanzig and R. D. Mountain, J. Chem. Phys. 43, 4464 (1965)

[79] A. Onuki, Phase Transition Dynamics (Cambridge University Press, Cambridge, 2002).

[80] In Ref. 14 with $N=10^{3}$ we calculated the time aver- age of $\left[\left\langle\sigma_{x y}\right\rangle(t)-\bar{\sigma}_{x y}\right]^{2}$ in plastic flow, denoted by $\mathcal{V}$. The $\mathcal{V}^{1 / 2}$ is the typical fluctuation amplitude. Comparison of the data in Ref. [14] and those in the present simulation with $N=9 \times 10^{3}$ (not shown here) indicates $\mathcal{V} \propto L^{-2}$. See Ref. 28 also for this point. Note that $L^{2} \mathcal{V}$ is equal to the time average of the quantity $L^{-2} \int d \boldsymbol{r}_{1} \int d \boldsymbol{r}_{2}\left\langle\delta \Pi_{x y}\left(\boldsymbol{r}_{1}, t\right) \delta \Pi_{x y}\left(\boldsymbol{r}_{2}, t\right)\right\rangle$ for the $x y$ component of the stress tensor $\Pi_{x y}(\boldsymbol{r}, t)\lfloor 79$.

[81] Y. Estrin and L.P. Kubin, Mater. Sci. Eng. A 137, 125 (1991); G. D'Anna and F. Nori, Phys. Rev. Lett. 85, 4096 (2000); P. Barat, A. Sarkar, P. Mukherjee, and S.K. Bandyopadhyay, Phys. Rev. Lett. 94, 055502 (2005)

[82] T. Shikata, H. Niwa, and Y. Morishima, J. Rheol. 42, 765 (1998).

[83] Dislocation gliding involves motions of a relatively small number of particles, while the other form of dislocation motions, climbing, requires motions of many particles and is not easily induced 3 .

[84] A.S. Argon and H.Y. Kuo, Mater. Sci. Eng. 39, 101 (1979).

[85] R. Peierls, Proc. Phys. Soc. 52, 34 (1940); F.R.N. Nabarro, ibid. 59, 256 (1947).

[86] M. Peach and J.S. Koehler, Phys. Rev. 80, 436 (1950). 